\title{
Pacific
}

Journal of

Mathematics

\section{ATTACHING HANDLES TO DELAUNAY NODOIDS}

FRANK PACARD AND HAROLD ROSENBERG 


\title{
ATTACHING HANDLES TO DELAUNAY NODOIDS
}

\author{
FRANK PACARD AND HAROLD ROSENBERG
}

\begin{abstract}
For all $m \in \mathbb{N}-\{0\}$, we prove the existence of a one-dimensional family of genus $m$, constant mean curvature (equal to 1 ) surfaces which are complete, immersed in $\mathbb{R}^{3}$, and have two Delaunay ends asymptotic to nodoidal ends. Moreover, these surfaces are invariant under the group of isometries of $\mathbb{R}^{3}$ leaving a horizontal regular polygon with $m+1$ sides fixed.
\end{abstract}

\section{Introduction}

Delaunay surfaces are complete, noncompact constant mean curvature surfaces of revolution in $\mathbb{R}^{3}$ which are either embedded or immersed. The embedded Delaunay surfaces are usually referred to as unduloids. The elements of this family are generated by roulettes of ellipses [Eells 1987], and they interpolate between a right cylinder $S^{1}\left(\frac{1}{2}\right) \times \mathbb{R} \subset \mathbb{R}^{3}$ and a singular surface which is constituted by infinitely many tangent spheres of radius 1 which are periodically arranged along the vertical axis. Close to the singular limit, the Delaunay unduloids can be understood as infinitely many spheres of radius 1 which are disjoint, arranged periodically along the vertical axis, each sphere being connected to its two nearest neighbors by catenoids, whose rotational axis is the vertical axis, which have been scaled by a small factor $\tau>0$.

The immersed Delaunay surfaces are referred to as nodoids. The elements of this family are generated by roulettes of hyperbolas [Eells 1987]. Again, part of this family converges to infinitely many spheres of radius 1 which are periodically arranged along the vertical axis. In contrast to unduloids, close to the singular limit, the Delaunay nodoids can be understood as infinitely many spheres of radius 1 which are either disjoint or slightly overlapping and which are arranged periodically along the vertical axis, each sphere being connected to its two nearest neighbors (with which it shares a slight overlap) by catenoids that have vertical axes and that have been scaled by a small factor $\tau>0$.

In this paper, we prove the existence of constant mean curvature surfaces which have two Delaunay ends (of nodoid type) and finite genus.

The first author is partially supported by the ANR-11-IS01-0002 grant.

MSC2010: primary 53C42; secondary 53A10.

Keywords: constant mean curvature surfaces, Delaunay surfaces. 
Theorem 1.1. For all $m \geq 1$, there exists a one parameter family of genus $m$ constant mean curvature (with mean curvature equal to 1) surfaces which are invariant under the action of the full dihedral group $\operatorname{Dih}_{m+1}^{(3)}$ (the group of isometries of $\mathbb{R}^{3}$ leaving a horizontal regular polygon with $m+1$ sides fixed) and which have two Delaunay ends asymptotic to nodoidal ends.

Let us briefly describe how these surfaces are constructed, since this will provide an opportunity to give a precise picture of the surfaces themselves.

As already mentioned, close to the singular limit, the Delaunay nodoids can be understood as infinitely many spheres of radius 1 which are either disjoint or slightly overlapping, arranged periodically along the vertical axis and which are connected together by catenoids, which have vertical axes, and which are scaled by a small factor $\tau>0$; these latter ones are called catenoidal necks. The spheres of radius 1 arranged along the vertical axis can be ordered (by the height of their centers) and can be indexed by $j \in \mathbb{Z}$ (without loss of generality, we can assume that the center of the sphere of index $j$ is at height $2 j+1)$. In this description, one can check that the distance between the centers of two consecutive spheres can be expanded as

$$
d_{\tau}=2+2 \tau \log \tau+\mathcal{O}(\tau)
$$

as $\tau$ tends to 0 . In order to obtain the surfaces of Theorem 1.1, instead of connecting the sphere indexed by 0 and the sphere indexed by 1 using one catenoidal neck, we connect these two spheres using $m+1$ catenoids which are scaled by a factor

$$
\tilde{\tau}=\frac{\tau}{m+1}+\mathcal{O}\left(\tau^{3 / 2}\right),
$$

and whose axes are vertical and pass through the vertices of a horizontal regular polygon (with $m+1$ sides) of size $\rho>0$. We show that this construction is successful provided the parameter $\rho$, which measures the size of the polygon, is carefully chosen (as a function of $\tau$ ), and, in fact, we find that

$$
\rho^{2}=\frac{m}{m+1} \frac{\tau}{2}+O\left(\tau^{5 / 4}\right)
$$

Notice that all the surfaces we construct have the same small vertical flux (we refer to Section 4 for a definition of the flux of a Delaunay surface).

Our construction is quite flexible and provides many other interesting constant mean curvature surfaces. For example, using similar ideas and proofs, one can also construct singly periodic constant mean curvature surfaces with (infinite) topology: starting with the spheres of radius 1 which are periodically arranged along the vertical axis and which are either disjoint or slightly overlapping, we can choose to connect any two consecutive spheres using $m+1$ catenoids scaled by a factor $\tilde{\tau}$ whose axes are vertical and pass through the vertices of a horizontal regular polygon (with 
$m+1$ sides) of size $\rho>0$. More generally, there is strong evidence for the following.

It should be possible to construct constant mean curvature surfaces starting from a subset $\mathfrak{Z} \subset \mathbb{Z}$ and assuming that, for all $j \in \mathbb{Z}-\mathfrak{Z}$, we decide to connect the sphere of index $j$ to the sphere of index $j+1$ using one catenoid whose axis is the vertical axis and which is scaled by a factor $\tau$, while, when $j \in \mathfrak{Z}$, we decide to connect the sphere of index $j$ to the sphere of index $j+1$ using $m+1$ catenoids whose axes are vertical and pass through the vertices of a small horizontal regular polygon (with $m+1$ sides) of size $\rho>0$ with

$$
\rho^{2} \sim \frac{m}{m+1} \frac{\tau}{2},
$$

and which are scaled by a factor $\tilde{\tau} \sim \tau /(m+1)$. We believe that this configuration can be perturbed into a genuine constant mean curvature surface.

We mention that the present construction is very much inspired by [Hauswirth and Pacard 2007], where the authors perform a construction of minimal surfaces in $\mathbb{R}^{3}$ that have finite genus and two Riemann type ends. In fact, part of the analysis in the present paper parallels the analysis in [Hauswirth and Pacard 2007]. Nevertheless, in the present situation, some extra technical difficulties arise in the construction (see Section 6), since the points where the connected sum is performed are located at the vertices of a polygon whose size tends to 0 as the parameter $\tau$ tends to 0 .

We end the introduction by giving an overview of the paper. In Section 2 we recall some well known facts about the mean curvature operator of normal graphs with special emphasize on the differential of the mean curvature operator. Section 3 is concerned with harmonic extensions on half cylinders, for which we prove some decay properties. Section 4 is quite long. It starts with a careful description of the Delaunay nodoids as the Delaunay parameter $\tau$ tends to 0 (that is, close to the singular limit). Then we proceed with the analysis of the Jacobi operator about a Delaunay surface as the Delaunay parameter tends to 0. Finally, starting on page 151, we apply the implicit function theorem about a half nodoid (which is a constant mean curvature surface with one boundary and one Delaunay end) to prove the existence of an infinite-dimensional family of constant mean curvature surfaces which have one Delaunay end and one boundary. These surfaces are close to the half nodoid we started with and are parametrized by their boundary data. In Section 6, we perform a similar analysis starting from the catenoid. As a result, we obtain the existence of an infinite-dimensional family of constant mean curvature surfaces which have two boundaries, are close to a truncated catenoid, and are parametrized by their boundary data. In Section 6, we start with a unit sphere, from which we excise one small disc close to the north pole and $m+1$ small discs arranged symmetrically at the vertices of a regular polygon near the south pole. We perturb this surface with $m+2$ boundaries applying the implicit function theorem 
to obtain an infinite-dimensional family of constant mean curvature surfaces which are parametrized by their boundary data. In the final section, we explain how all these pieces can be connected together to produce the surfaces in Theorem 1.1. At this stage, the problem then reduces to being able to choose the boundary data of the different summands so that their union is a $\mathscr{b}^{1}$ surface, since elliptic regularity theory will imply that what we have built is a smooth surface of constant mean curvature.

The construction relies heavily on the analysis of elliptic operators on noncompact spaces as in [Melrose 1993; Mazzeo 1991; Lockhart and McOwen 1985]. It is true that similar techniques and ideas have already been used in many constructions, but the proofs are usually hard to read for nonspecialists, since they always refer to results which are difficult to find in the literature in the precise form they are needed. This is why we have decided to present complete proofs based on simple well-known tools; we hope that this will help the interested reader master these techniques.

Finally, we mention a problem related to our work. To introduce this problem, we consider $\Sigma$ to be the union of the upper hemisphere of the sphere of radius 1 centered at the points $(0,0,-1)$ and the lower hemisphere of the sphere of radius 1 centered at the points $(0,0,1)$. The existence of unduloids, nodoids with small Delaunay parameters, and the surfaces we construct in this paper shows that, for all $\epsilon>0$, there exist infinitely many surfaces of constant mean curvature 1 that are included in an $\epsilon$-tubular neighborhood of the unduloid and are not congruent. Obviously a similar result holds for the surface $\Sigma$.

Now, if we consider two radius 1 spheres tangent at a point, can we find constant mean curvature $(=1)$ surfaces (with no boundary) in any small tubular neighborhood of this configuration? In fact, we can not even answer the following (apparently) simpler but striking question: is there any compact mean curvature $(=1)$ surface (with no boundary) near a radius 1 sphere? More precisely, is there an $\epsilon_{0}>0$ such that if $\Sigma$ is a mean curvature $(=1)$ surface in the $\epsilon_{0}$-tubular neighborhood of a radius 1 sphere, is $\Sigma$ congruent to the sphere? In other words, what is the form of a compact constant mean curvature surface?

\section{Generalities}

The mean curvature. We gather some basic material concerning the mean curvature of a surface in Euclidean space. All these results are well known, but we feel that collecting them here makes the paper easier to read. This also gives us an opportunity to introduce some of the notation we use throughout the paper. We refer to [Colding and Minicozzi 2011; Lawson 1977] for further details.

Let us assume that $\Sigma$ is a surface embedded in $\mathbb{R}^{3}$. We denote by $g$ the metric induced on $\Sigma$ by the Euclidean metric $\stackrel{\circ}{g}$, and by $h$ the second fundamental form, 
defined by

$$
h\left(t_{1}, t_{2}\right)=-\stackrel{\circ}{g}\left(\nabla_{t_{1}} N, t_{2}\right)
$$

for all $t_{1}, t_{2} \in T \Sigma$. Here $N$ is a unit normal vector field on $\Sigma$. In this paper, we agree that the mean curvature of a surface is defined to be the average of the principal curvatures, or, since we are interested in 2-dimensional surfaces, the half of the trace of the second fundamental form. Hence the mean curvature of $\Sigma$ is given by

$$
H:=\frac{1}{2} \operatorname{tr}^{g} h,
$$

and the mean curvature vector is then given by $\vec{H}:=H N$.

For computational purposes, we recall that the mean curvature appears in the first variation of the area functional. More precisely, given $w$, a sufficiently small smooth function which is defined on $\Sigma$ and has compact support, we consider the surface $\Sigma_{w}$, which is the normal graph over $\Sigma$ for the function $w$. Namely,

$$
\Sigma \ni p \mapsto p+w(p) N(p) \in \Sigma_{w} .
$$

We denote by $A_{w}$ the area of the surface $\Sigma_{w}$ (we assume that this area is finite). Then

$$
D A_{\mid w=0}(v)=-2 \int_{\Sigma} H v d \operatorname{vol}_{g}
$$

In the case where surfaces close to $\Sigma$ are parametrized as graphs over $\Sigma$ using a vector field $\tilde{N}$ which is transverse to $\Sigma$ but which is not necessarily a unit normal vector field, the previous formula has to be modified. Let us denote by $\tilde{\Sigma}_{w}$ the surface which is the graph over $\Sigma$, using the vector field $\tilde{N}$, for some sufficiently small smooth function $w$. Namely,

$$
\Sigma \ni p \mapsto p+w(p) \tilde{N}(p) \in \tilde{\Sigma}_{w} .
$$

We denote by $\tilde{A}_{w}$ the area of this surface. The previous formula must be changed to

$$
D \tilde{A}_{\mid w=0}(v)=-2 \int_{\Sigma}(\vec{H} \cdot \tilde{N}) v d \operatorname{vol}_{g} .
$$

In the next result, we give the expression of the mean curvature $H_{w}$ of the surface $\Sigma_{w}$ in terms of $w$. Some notation is needed. For $z \in \mathbb{R}$ small enough, we define $g_{z}$ to be the induced metric on the parallel surface

$$
\Sigma_{z}:=\Sigma+z N .
$$

It is given explicitly by

$$
g_{z}=g-2 z h+z^{2} k,
$$

where the tensor $k$ is defined by

$$
k\left(t_{1}, t_{2}\right):=g\left(\nabla_{t_{1}} N, \nabla_{t_{2}} N\right)
$$

for all $t_{1}, t_{2} \in T \Sigma$. 
Proposition 2.1. The mean curvature $H_{w}$ of the surface $\Sigma_{w}$ is given by the formula

$$
\begin{aligned}
H_{w}=\frac{1}{2} \sqrt{1+\left|\nabla^{g_{w}} w\right|^{2}} \operatorname{tr}^{g}(h-w k)+ & \frac{1}{2} \operatorname{div}_{g_{w}}\left(\frac{\nabla^{g w} w}{\sqrt{1+\left|\nabla^{g_{w}} w\right|^{2}}}\right) \\
& -\frac{1}{2} \frac{1}{\sqrt{1+\left|\nabla^{g_{w}} w\right|}}(h-w k)\left(\nabla^{g_{w}} w, \nabla^{g_{w}} w\right) .
\end{aligned}
$$

Proof. The induced metric $\tilde{g}$ on $\Sigma_{w}$ is given by

$$
\tilde{g}=g_{z=w}+d w \otimes d w .
$$

This implies that

$$
\operatorname{det} \tilde{g}=\left(1+\left|\nabla^{g_{w}} w\right|^{2}\right) \operatorname{det} g_{w} .
$$

We can now compute the area of $\Sigma_{w}$,

$$
A_{w}=\int_{\Sigma} \sqrt{1+\left|\nabla^{g_{w}} w\right|^{2}} d \operatorname{vol}_{g_{w}},
$$

as well as the differential of this functional with respect to $w$. In doing so, one should be careful that the function $w$ appears implicitly in the definition of $g_{w}$. Using integration by parts, we find

$$
\begin{aligned}
D A_{w}(v)= & -\int_{\Sigma} \operatorname{div}_{g_{w}}\left(\frac{\nabla^{g_{w}} w}{\sqrt{1+\left|\nabla^{g_{w}} w\right|^{2}}}\right) v d \operatorname{vol}_{g_{w}} \\
& -\frac{1}{2} \int_{\Sigma} \frac{g_{w}^{\prime}\left(\nabla^{g_{w}} w, \nabla^{g_{w}} w\right)}{\sqrt{1+\left|\nabla^{g_{w}} w\right|^{2}}} v d \operatorname{vol}_{g_{w}}+\frac{1}{2} \int_{\Sigma} \sqrt{1+\left|\nabla^{g_{w}} w\right|^{2}} \operatorname{tr}^{g_{w}} g_{w}^{\prime} v d \operatorname{vol}_{g_{w}},
\end{aligned}
$$

where $g_{w}^{\prime}:=\partial_{z} g_{z \mid z=w}=-2(h-w k)$. To proceed, observe that, if $N_{w}$ denotes the unit normal vector field about $\Sigma_{w}$, we have

$$
N_{w}=\frac{1}{\sqrt{1+\left|\nabla^{g_{w}} w\right|^{2}}}\left(N-\nabla^{g_{w}} w\right)
$$

and hence we get

$$
d \operatorname{vol}_{g_{w}}=\left(N_{w} \cdot N\right) d \operatorname{vol}_{\tilde{g}} .
$$

The result then follows at once from (2-1).

Linearized mean curvature operators. Again, the material in this section is well known, and we refer to [Colding and Minicozzi 2011; Lawson 1977] for a more detailed description. The Jacobi operator appears in the linearization of the mean curvature operator when nearby surfaces are parametrized as normal graphs over a given surface. Indeed, we can consider the nonlinear operator $w \mapsto H_{w}$, which is defined, for example, from the space $\mathscr{C}_{\mathrm{loc}}^{2}(\Sigma)$ into the space $\mathscr{C}_{\mathrm{loc}}^{0}(\Sigma)$, and it 
follows from Proposition 2.1 that the differential of this operator with respect to $w$, computed at $w=0$, is given by

$$
J:=D H_{w=0}=\frac{1}{2}\left(\Delta_{g}+\operatorname{tr}^{g} k\right),
$$

where $\Delta_{g}$ is the Laplace-Beltrami operator on $\Sigma$ and $\operatorname{tr}^{g} k$ is the square of the norm of the shape operator.

Finally, we recall that if $\Sigma$ is a constant mean curvature surface and if $\Xi$ is a killing vector field (that is, $\Xi$ generates a one parameter family of isometries), the function $N \cdot \Xi$, which is usually referred to as a Jacobi field, satisfies

$$
J(N \cdot \Xi)=0 .
$$

This is probably a good time to recall some elementary facts concerning linearized mean curvature operators when different vector fields are used. As above, we assume that we are given a vector field $\tilde{N}$ which is transverse to $\Sigma$, but which is not necessarily a unit normal vector field. Any surface close enough to $\Sigma$ can be considered either as a normal graph over $\Sigma$ or as a graph over $\Sigma$, using the vector field $\tilde{N}$. Hence we can define two nonlinear operators

$$
w \mapsto H_{w} \quad \text { and } \quad w \mapsto \tilde{H}_{w},
$$

which are (respectively) the mean curvature of the normal graph of $w$ and the mean curvature of the graph of $w$ using the vector field $\tilde{N}$. The following result gives the relation between the differentials of these two operators at $w=0$.

Proposition 2.2 [Mazzeo et al. 2001]. The relation

$$
D \tilde{H}_{\mid w=0}(v)=D H_{\mid w=0}((\tilde{N} \cdot N) v)+(\nabla H \cdot \tilde{N}) v
$$

holds for any $v \in \mathscr{C}_{\text {loc }}^{2}(\Sigma)$, where $H$ denotes the mean curvature of $\Sigma$. In the particular case where $\Sigma$ has constant mean curvature, this formula reduces to

$$
D \tilde{H}_{\mid w=0}(v)=D H_{\mid w=0}((\tilde{N} \cdot N) v) .
$$

Proof. The implicit function theorem can be applied to the equation

$$
p+t N(p)=q+s \tilde{N}(q)
$$

to express (at least locally) $p$ and $t$ as functions of $q$ and $s$, namely,

$$
p=\Phi(q, s) \text { and } t=\Psi(q, s),
$$

with $\Phi(q, 0)=q$ and $\Psi(q, 0)=0$. It is easy to check that

$$
\partial_{s} \Phi(\cdot, 0)=\tilde{N}^{T} \quad \text { and } \quad \partial_{s} \Psi(\cdot, 0)=\tilde{N} \cdot N,
$$

where superscript $T$ denotes the projection over $T \Sigma$. 
Differentiating the identity

$$
H_{\Psi(\cdot, w)}(\Phi(q, w(q)))=\tilde{H}_{w}(q)
$$

with respect to $w$, at $w=0$, we find

$$
D H_{\mid w=0}\left(\partial_{s} \Psi(\cdot, 0) v\right)+\nabla H_{\mid w=0} \cdot \partial_{s} \Phi v=D \tilde{H}_{\mid w=0}(v) .
$$

The result then follows from the expression of $\partial_{S} \Phi$ and $\partial_{S} \Psi$ and the fact that $\tilde{H}_{\mid w=0}=H_{\mid w=0}$.

\section{Harmonic extensions}

For all $x \in \mathbb{R}^{2}$ and all $r>0$ we denote by $D(x, r) \subset \mathbb{R}^{2}$ the open disc of radius $r$ centered at $x$ and by $\bar{D}(x, r) \subset \mathbb{R}^{2}$ the closed disc of radius $r$ centered at $x$. In this section, we study the harmonic extension of a function which is defined on the unit circle $S^{1}$ to a half cylinder $[0, \infty) \times S^{1}$, or to a punctured disc $\bar{D}^{*}(0,1)$, or to the complement of the closed unit disc $\mathbb{R}^{2}-D(0,1)$. We use the fact that all these domains are conformal to each other and that the Laplacian is conformally invariant in dimension 2.

Let us assume that we are given a function $f \in \mathscr{C}^{2, \alpha}\left(S^{1}\right)$. We consider $F$ to be the bounded harmonic extension of $f$ to the half cylinder, endowed with the cylindrical metric

$$
g_{\text {cyl }}=d s^{2}+d \theta^{2} .
$$

In other words, $F$ is bounded and is a solution of

$$
\Delta_{g_{\mathrm{cyl}}} F=0
$$

in $[0, \infty) \times S^{1}$ with $F=f$ on $\{0\} \times S^{1}$.

Observe that one can use cylindrical coordinates to parametrize the punctured unit disc by

$$
\tilde{X}(s, \theta)=\left(e^{-s} \cos \theta, e^{-s} \sin \theta\right),
$$

in which case the function $\tilde{F}$ defined by $\tilde{F} \circ \tilde{X}:=F$ is the unique bounded solution of

$$
\Delta \tilde{F}=0
$$

(where $\Delta$ denotes the Laplacian in $\mathbb{R}^{2}$ ) in the punctured unit disc with $\tilde{F}=f$ on $S^{1}$. We set

$$
W_{f}^{\text {ins }}:=\tilde{F} .
$$

Also, one can use cylindrical coordinates to parametrize the complement of the unit disc in $\mathbb{R}^{2}$ by

$$
\hat{X}(s, \theta)=\left(e^{s} \cos \theta, e^{s} \sin \theta\right),
$$


in which case $\hat{F}$, defined by $\hat{F} \circ \hat{X}=F$, is the unique bounded solution of

$$
\Delta \hat{F}=0
$$

in the complement of the unit disc with $\hat{F}=f$ on $S^{1}$. We set

$$
W_{f}^{\text {out }}:=\hat{F} \text {. }
$$

All properties of $F$ will transfer easily to $\tilde{F}$ and $\hat{F}$.

Given a function $f$ defined on $S^{1}$, we shall frequently assume that one or both of the following assumptions are fulfilled:

$$
\begin{gathered}
\int_{S^{1}} f d \theta=0 . \\
\int_{S^{1}} \cos \theta f d \theta=\int_{S^{1}} \sin \theta f d \theta=0 .
\end{gathered}
$$

The following result follows essentially from [Fakhi and Pacard 2000], where a similar result was proven in higher dimensions.

Lemma 3.1. There exists a constant $C>0$ such that, for all $f \in \mathscr{C}^{2, \alpha}\left(S^{1}\right)$ satisfying (H1), we have

$$
\left\|e^{S} F\right\|_{\mathscr{C}^{2, \alpha}\left([0, \infty) \times S^{1}\right)} \leq C\|f\|_{\mathscr{C}^{2, \alpha}\left(S^{1}\right)},
$$

and, if $f$ satisfies $(\mathrm{H} 1)$ and $(\mathrm{H} 2)$, we have

$$
\left\|e^{2 s} F\right\|_{\mathscr{C}^{2, \alpha}\left([0, \infty) \times S^{1}\right)} \leq C\|f\|_{\mathscr{C}^{2, \alpha}\left(S^{1}\right)} .
$$

Before we proceed with the proof of this result, let us emphasize that the norms in $\mathscr{C}^{2, \alpha}\left([0, \infty) \times S^{1}\right)$ are computed with respect to the cylindrical metric $g_{\text {cyl }}$.

Proof. We consider the Fourier series decomposition of the function $f$

$$
f(\theta)=\sum_{n \in \mathbb{Z}} f_{n} e^{i n \theta} .
$$

Observe that $f_{0}=0$ when (H1) is fulfilled and $f_{ \pm 1}=0$ when (H2) is fulfilled. For the time being, let us assume that both (H1) and (H2) are satisfied. Then the (bounded) harmonic extension of $f$ is given explicitly by

Since

$$
F(s, \theta)=\sum_{|n| \geq 2} e^{-|n| s} f_{n} e^{i n \theta} .
$$

$$
\left|f_{n}\right| \leq\|f\|_{L^{\infty}\left(S^{1}\right)},
$$

we get the pointwise estimate

$$
|F(s, \theta)| \leq 2\|f\|_{L^{\infty}\left(S^{1}\right)} \sum_{n \geq 2} e^{-n s} \leq 2\|f\|_{L^{\infty}\left(S^{1}\right)} \frac{e^{-2 s}}{1-e^{-s}},
$$


which implies that

$$
\sup _{[1, \infty) \times S^{1}} e^{2 s}|F(s, \theta)| \leq C\|f\|_{L^{\infty}\left(S^{1}\right)} .
$$

Increasing the value of $C>0$ if necessary, we can use the maximum principle in the annular region $[0,1] \times S^{1}$ to get

$$
\sup _{[0, \infty) \times S^{1}} e^{2 s}|F(s, \theta)| \leq C\|f\|_{L^{\infty}\left(S^{1}\right)} .
$$

The estimates for the derivatives of $F$ then follow from classical elliptic estimates, since Schauder's estimates can be applied on each annulus $[s, s+1] \times S^{1}$ for all $s \geq 0$. This already completes the proof of the result when both (H1) and (H2) are fulfilled. When only (H1) holds, one has to take into account the function $f_{ \pm 1} e^{-s} e^{ \pm i \theta}$, which accounts for the slower decay of $F$ as $e^{-s}$.

\section{The Delaunay nodoids}

Parametrization and notations. The Delaunay nodoid $\mathfrak{D}_{\tau}$ is a surface of revolution which can be parametrized by

$$
X_{\tau}(s, \theta):=\left(\phi_{\tau}(s) \cos \theta, \phi_{\tau}(s) \sin \theta, \psi_{\tau}(s)\right),
$$

where $(s, \theta) \in \mathbb{R} \times S^{1}$. Here the functions $\phi_{\tau}$ and $\psi_{\tau}$ depend on the real parameter $\tau>0$, but, unless necessary, we do not make this apparent in the notation. The function $\phi$ is chosen to be the unique, smooth, periodic, nonconstant solution of

$$
\dot{\phi}^{2}+\left(\phi^{2}-\tau\right)^{2}=\phi^{2},
$$

which takes its minimum value at $s=0$ (we denote by $\cdot$ differentiation with respect to the parameter $s$ ), and the function $\psi$ is obtained by integration of

$$
\dot{\psi}=\phi^{2}-\tau
$$

with initial condition $\psi(0)=0$. Observe that $\phi$ is a smooth solution of

$$
\ddot{\phi}+2 \phi\left(\phi^{2}-\tau\right)=\phi .
$$

Since $\phi^{2}-\tau$ changes sign, the function $\psi$ is not monotone, and closer inspection of the solutions shows that $\mathfrak{D}_{\tau}$ is actually not embedded. The Delaunay nodoids also arise as the surface of revolution whose generating curve is a roulette of a hyperbola; we refer to [Eells 1987] for a description of this construction. The quantity $(1 / 4) \tau$ is sometimes referred to as the vertical flux of the Delaunay surface $\mathfrak{D}_{\tau}$; see [Rossman 2005, Definition 3.1]. Define

$$
\underline{\tau}:=\frac{\sqrt{1+4 \tau}-1}{2} \text { and } \bar{\tau}:=\frac{\sqrt{1+4 \tau}+1}{2},
$$


which, thanks to (4-2) and (4-4), are, respectively, the minimum and maximum values of $\phi$. As already mentioned, the function $\phi$ is periodic. We agree that $s_{\tau}$ denotes one half of the fundamental period of $\phi$. Using (4-2), we can write

$$
s_{\tau}=\int_{\underline{\tau}}^{\bar{\tau}} \frac{d \zeta}{\sqrt{\zeta^{2}-\left(\zeta^{2}-\tau\right)^{2}}} .
$$

In the above parametrization, the induced metric on $\mathfrak{D}_{\tau}$ is given by

$$
g_{\tau}:=\phi^{2}\left(d s^{2}+d \theta^{2}\right),
$$

and it is easy to check that the second fundamental form on $\mathfrak{D}_{\tau}$ is given by

$$
h_{\tau}:=\left(\phi^{2}+\tau\right) d s^{2}+\left(\phi^{2}-\tau\right) d \theta^{2},
$$

when the unit normal vector field is chosen to be

$$
N_{\tau}:=\frac{1}{\phi}\left(\left(\tau-\phi^{2}\right) \cos \theta,\left(\tau-\phi^{2}\right) \sin \theta, \dot{\phi}\right) .
$$

Finally, the tensor $k_{\tau}$ is given by

$$
k_{\tau}:=\left(\phi+\frac{\tau}{\phi}\right)^{2} d s^{2}+\left(\phi-\frac{\tau}{\phi}\right)^{2} d \theta^{2} .
$$

In particular, the formulae for the induced metric and the second fundamental form imply that the mean curvature of this surface is constant and equal to

$$
H:=\frac{1}{2} \operatorname{tr}^{g} h=1 .
$$

In these coordinates, it follows at once from the expression of $g_{\tau}$ and $k_{\tau}$ that the Jacobi operator about $\mathfrak{D}_{\tau}$ is given by

$$
J_{\tau}:=\frac{1}{2 \phi^{2}}\left(\partial_{s}^{2}+\partial_{\theta}^{2}+2\left(\phi^{2}+\frac{\tau^{2}}{\phi^{2}}\right)\right) .
$$

Structure and refined asymptotics. The structure of the Delaunay surfaces $\mathfrak{D}_{\tau}$ is well understood, and it is known that, as the parameter $\tau$ tends to $0, \mathfrak{D}_{\tau}$ converges to the union of infinitely many spheres of radius 1 which are arranged periodically along the vertical axis. To get a better grasp on the structure of $\mathfrak{D}_{\tau}$ as $\tau$ tends to 0 , we have the following results, which were already used in many constructions of constant mean curvature surfaces by gluing [Mazzeo and Pacard 2001; Mazzeo et al. 2001; 2005]. For completeness we give independent proofs of these results.

Lemma 4.1. As $\tau$ tends to 0 , the following holds.

(i) The sequence of functions $\phi_{\tau}\left(\cdot+s_{\tau}\right)$ converges uniformly on compact sets of $\mathbb{R}$ to the function $s \mapsto(\cosh s)^{-1}$. 
(ii) The sequence of functions $\psi_{\tau}\left(\cdot+s_{\tau}\right)-\psi_{\tau}\left(s_{\tau}\right)$ converges uniformly on compact sets of $\mathbb{R}$ to the function $s \mapsto \tanh s$.

Proof. It is easy to check that $\phi_{\tau}\left(\cdot+s_{\tau}\right)$ is even and that $\phi_{\tau}\left(s_{\tau}\right)=\bar{\tau}$ converges to 1 as $\tau$ tends to 0 (this follows from the fact that the function $\phi_{\tau}$ achieves its maximum value when $s=s_{\tau}$ ). Passing to the limit in (4-2), we conclude that the sequence of functions $\phi_{\tau}$ converges uniformly on compact sets of $\mathbb{R}$ to a function $\phi_{0}$ which is a solution of

$$
\ddot{\phi}_{0}+2 \phi_{0}^{3}=\phi_{0} .
$$

Moreover, the function $\phi_{0}$ is even and is equal to 1 when $s=0$. Therefore, necessarily $\phi_{0}(s)=(\cosh s)^{-1}$. Next, one can pass to the limit in (4-3) to prove that the sequence $\psi_{\tau}\left(\cdot+s_{\tau}\right)-\psi_{\tau}\left(s_{\tau}\right)$ converges to a function $\psi_{0}$ which is a solution of

$$
\dot{\psi}_{0}=\phi_{0}^{2}
$$

and satisfies $\psi_{0}(0)=0$. We find that $\psi_{0}(s)=\tanh s$.

Now we investigate the behavior of $\mathfrak{D}_{\tau}$ close to the origin in $\mathbb{R}^{3}$. It turns out that the sequence of rescaled surfaces $(1 / \tau) \mathfrak{D}_{\tau}$ converges on compact sets of $\mathbb{R}^{3}$ to a catenoid whose axis is the vertical axis:

Lemma 4.2. As $\tau$ tends to 0 , the following holds:

(i) The sequence of functions $(1 / \tau) \phi_{\tau}$ converges uniformly on compact sets of $\mathbb{R}$ to the function $s \mapsto \cosh s$.

(ii) The sequence of functions $(1 / \tau) \psi_{\tau}$ converges uniformly on compact sets of $\mathbb{R}$ to the function $s \mapsto-s$.

Proof. It is easy to check that $\phi_{\tau}$ is even and that $(1 / \tau) \phi_{\tau}(0)=\underline{\tau} / \tau$ converges to 1 as $\tau$ tends to 0 (this follows from the fact that the function $\phi_{\tau}$ achieves its minimum value when $s=0)$. Passing to the limit in (4-2), we conclude that $(1 / \tau) \phi_{\tau}$ converges uniformly on compact sets of $\mathbb{R}$ to a function $\phi_{0}$ which is a solution of

$$
\ddot{\phi}_{0}=\phi_{0} \text {. }
$$

Moreover, $\phi_{0}$ is even and is equal to 1 when $s=0$. Therefore, $\phi_{0}(s)=\cosh s$. Next, one can pass to the limit in (4-3) to prove that the sequence $(1 / \tau) \psi_{\tau}$ converges to a function $\psi_{0}$ that is a solution of $\dot{\psi}_{0}=-1$ and satisfies $\psi_{0}(0)=0$. Therefore, we conclude that $\psi_{0}(s)=-s$, as desired.

Geometrically, these results show that, as $\tau$ tends to 0 , the Delaunay surface $\mathfrak{D}_{\tau}$ is close to infinitely many spheres of radius 1 which are arranged along the vertical axis (and are slightly overlapping), each sphere connected to its two neighbors by small rescaled catenoids. 
We will need a refined and more quantitative version of Lemma 4.2. Observe that $\dot{\phi}<0$ in $\left(-s_{\tau}, 0\right)$, and hence $\phi$ is a diffeomorphism from $\left(-s_{\tau}, 0\right)$ into $(\underline{\tau}, \bar{\tau})$. We can define the change of variables

$$
r=\phi_{\tau}(s),
$$

to express $s \in\left(-s_{\tau}, 0\right)$ as a function of $r \in(\underline{\tau}, \bar{\tau})$, so that the equality

$$
X_{\tau}(s, \theta)=\left(r \cos \theta, r \sin \theta, u_{\tau}(r)\right),
$$

where $r=\phi_{\tau}(s)$, holds for some function $u_{\tau}$ defined in an annulus of $\mathbb{R}^{2}$. Geometrically, this means that the image of $\left(-s_{\tau}, 0\right) \times S^{1}$ by $X_{\tau}$ is a vertical graph for some function $u_{\tau}$ which is defined over the annulus

$$
\left\{x \in \mathbb{R}^{2}: \underline{\tau}<|x|<\bar{\tau}\right\} .
$$

Proposition 4.3. As $\tau$ tends to 0 ,

$$
u_{\tau}(r)=\frac{\tau}{\sqrt{1+2 \tau}} \log \frac{2 r}{\tau}+\mathcal{O}_{\mathscr{C} \infty}\left(\frac{\tau^{3}}{r^{2}}\right)+\mathcal{O}_{\mathscr{C} \infty}\left(r^{2}\right)
$$

for $r \in\left(2 \underline{\tau}, \frac{1}{2} \bar{\tau}\right)$ uniformly as $\tau$ tends to 0 .

The notation $f_{1}=0_{\mathscr{C} \infty}\left(f_{2}\right)$ means that the function $f_{1}$ and all its derivatives with respect to the vector fields $r \partial_{r}$ and $\partial_{\theta}$ are bounded by a constant (depending on the order of derivation) times the (positive) function $f_{2}$.

Proof. By definition $\underline{\tau}$ is the minimum value of $\phi$. Hence we can write

$$
\phi(s)=\underline{\tau} \cosh (w(s))
$$

for some function $w$ which vanishes at $s=0$. Plugging this into (4-2), we find that the function $w$ is a solution of

$$
\dot{w}^{2}=1+2 \tau-\underline{\tau}^{2}\left(1+\cosh ^{2} w\right) .
$$

As long as $|w(s)-s| \leq 1$, we can estimate

$$
w(s)=\sqrt{1+2 \tau} s+\mathcal{O}\left(\tau^{2} \cosh ^{2} s\right) .
$$

In particular, we conclude a posteriori that $|w(s)-s| \leq 1$ holds, and hence that the above estimate is justified, provided $|s| \leq-\log \tau-c$ for some constant $c>0$ independent of $\tau \in(0,1)$. In the range of study, we are entitled to consider the change of variable

$$
r=\underline{\tau} \cosh w(s),
$$

and express $s<0$ as a function of $r$. We find

$$
\sqrt{1+2 \tau} s=-\log \frac{2 r}{\underline{\tau}}+\mathcal{O}\left(\frac{\tau^{2}}{r^{2}}\right)+\mathcal{O}\left(r^{2}\right)
$$


Finally, using (4-3), we can write

$$
\dot{\psi}=-\tau+\underline{\tau}^{2} \cosh ^{2} w .
$$

Integrating over $s$, we get

$$
\psi(s)=-\tau s+\mathcal{O}\left(\tau^{2} \cosh ^{2} s\right),
$$

and the result follows directly from (4-7) together with the fact that $u_{\tau}(r)=\psi(s)$, by definition, Similar estimates can be obtained for the derivatives of $u_{\tau}$.

A close inspection of the proof of Proposition 4.3 also yields the following.

Lemma 4.4. As $\tau$ tends to 0 , half of the fundamental period of the function $\phi_{\tau}$ can be expanded as

$$
s_{\tau}=-\log \tau+\mathscr{O}(1),
$$

and there exists a constant $C>1$ such that

$$
\frac{\tau}{C} \cosh s \leq \phi_{\tau} \leq C \tau \cosh s,
$$

when $s \in\left(-s_{\tau}, s_{\tau}\right)$, this estimate being uniform as $\tau$ tends to 0 .

Proof. The asymptotics of the half period of $\phi$ can also be derived from the formula (4-6). The estimate for $\phi$ follows from the proof of Proposition 4.3.

Analysis of the Jacobi operator. We analyze the mapping properties of the Jacobi operator about the Delaunay surface $\mathfrak{D}_{\tau}$, paying special attention to what happens when $\tau$ tends to 0 . This analysis is very close to the one available in [Mazzeo and Pacard 2001; Hauswirth and Pacard 2007]. Again, we give a self-contained proof adapted to the nonlinear argument we use in subsequent sections.

We first analyze the behavior, as $\tau$ tends to 0 , of the potential which appears in the expression of $J_{\tau}$. To this end, we assume that, for each $\tau>0$, we are given a real number $t_{\tau} \in \mathbb{R}$ and we define

$$
\xi_{\tau}:=\left(\phi_{\tau}^{2}+\frac{\tau^{2}}{\phi_{\tau}^{2}}\right)\left(\cdot-t_{\tau}\right) .
$$

Lemma 4.5. As $\tau$ tends to 0 , a subsequence of the sequence of functions $\xi_{\tau}$ converges uniformly on compact sets of $\mathbb{R}$ either to 0 or to the function $s \mapsto$ $\left(\cosh \left(s-s_{0}\right)\right)^{-2}$, for some $s_{0} \in \mathbb{R}$.

Proof. We define

$$
\zeta_{\tau}:=\left(\phi_{\tau}+\frac{\tau}{\phi_{\tau}}\right)\left(\cdot-t_{\tau}\right) .
$$

Using (4-2), we find that $\zeta_{\tau}$ is a solution of

$$
\dot{\zeta}_{\tau}^{2}=\left(\zeta_{\tau}^{2}-2 \tau\right)\left(1+4 \tau-\zeta_{\tau}^{2}\right)
$$


and, additionally, (4-4) implies that

$$
\ddot{\zeta}_{\tau}=\zeta_{\tau}\left(1+6 \tau-2 \zeta_{\tau}^{2}\right)
$$

Now we can estimate

$$
\zeta_{\tau}^{2}=\left(\phi-\frac{\tau}{\phi}\right)^{2}+4 \tau \leq 1+4 \tau,
$$

where we have used (4-2), which provides the estimate $\left(\phi^{2}-\tau\right)^{2} \leq \phi^{2}$. This implies that $\zeta_{\tau}$ and its derivatives remain bounded as $\tau$ tends to 0 . We can then let $\tau$ tend to 0 and pass to the limit in (4-8) and (4-9) to get that, as $\tau$ tends to 0 , the sequence $\zeta_{\tau}$ converges on compact sets to a solution of the equation $\ddot{\zeta}=\zeta\left(1-2 \zeta^{2}\right)$, which satisfies $\dot{\zeta}^{2}=\zeta^{2}\left(1-\zeta^{2}\right)$. Hence $\zeta$ is either 0 or a translation of $z \mapsto(\cosh s)^{-1}$. The result then follows from the identity $\xi_{\tau}=\zeta_{\tau}^{2}-2 \tau$.

We denote by $\pm \delta_{j}(\tau)$, for $j \in \mathbb{N}$, the indicial roots of the operator $J_{\tau}$. Recall that the indicial roots $\pm \delta_{j}$ correspond to the indicial roots of

$$
J_{\tau, j}:=\frac{1}{2 \phi^{2}}\left(\partial_{t}^{2}-j^{2}+2\left(\phi^{2}+\frac{\tau^{2}}{\phi^{2}}\right)\right),
$$

which appears in the Fourier decomposition of the operator $J_{\tau}$ in the $\theta$ variable. The indicial roots of $J_{\tau, j}$ characterize the exponential growth or decay rate at infinity of the solutions of the homogeneous problem $J_{\tau, j} w=0$. In general, it is a very hard problem to determine the exact value of the indicial roots of an operator, but in the present case, taking advantage of the geometric nature of the problem, we can prove the following.

Proposition 4.6. For all $\tau>0$, we have $\delta_{0}(\tau)=\delta_{1}(\tau)=0$. Furthermore, for $j \geq 2$,

$$
\delta_{j}(\tau) \geq \sqrt{j^{2}-2-4 \tau},
$$

provided $\tau<\sqrt{j^{2}-2}$.

Proof. The fact that $\delta_{0}(\tau)=0$ follows from the observation that the function $\dot{\phi} / \phi$ is periodic and solves

$$
J_{\tau, 0}(\dot{\phi} / \phi)=0 .
$$

This follows from direct computation, or it can be derived from the fact that

$$
\Phi_{0}^{+}:=\dot{\phi} / \phi
$$

is the Jacobi field associated to vertical translation (see page 134). Since the function $\phi$ is periodic, the homogeneous problem $J_{\tau, 0} w=0$ has a bounded solution, which implies that $\delta_{0}(\tau)=0$. 
The fact that $\delta_{1}(\tau)=0$ follows from the observation that the function $\phi-\tau / \phi$ is periodic and solves

$$
J_{\tau, 1}\left(\phi-\frac{\tau}{\phi}\right)=0
$$

Again, this follows from direct computation or can be derived from the fact that

$$
\Phi_{1}^{+}:=\left(\phi-\frac{\tau}{\phi}\right) \cos \theta \quad \text { and } \quad \Phi_{-1}^{+}:=\left(\phi-\frac{\tau}{\phi}\right) \sin \theta
$$

are the Jacobi fields associated to translations perpendicular to the axis of the Delaunay surface.

The estimate from below for the other indicial roots follows from the fact that, according to (4-2),

$$
2\left(\phi^{2}+\frac{\tau^{2}}{\phi^{2}}\right)=2\left(\phi-\frac{\tau}{\phi}\right)^{2}+4 \tau \leq 2+4 \tau .
$$

This, in particular, implies that the potential in $\partial_{s}^{2}-j^{2}+2\left(\phi^{2}+\tau^{2} / \phi^{2}\right)$ can be estimated from below by $\bar{\delta}_{j}^{2}$, where

$$
\bar{\delta}_{j}:=\sqrt{j^{2}-2-4 \tau} .
$$

The result then follows from the maximum principle and standard ODE arguments, since the function $s \mapsto e^{\bar{\delta}_{j} s}$ can be used as a barrier to prove the existence of two positive solutions of $J_{\tau, j} w=0$ which are defined on $(0, \infty)$, one being bounded from above by $e^{-\bar{\delta}_{j} s}$ and the other from below by $e^{\bar{\delta}_{j} s}$. This implies that $\delta_{j} \geq \bar{\delta}_{j}$, completing the proof of the result.

For all $\delta \in \mathbb{R}$, we define the operator

$$
\begin{aligned}
L_{\delta}: e^{\delta s} \mathscr{C}^{2, \alpha}\left(\mathbb{R} \times S^{1}\right) & \rightarrow e^{\delta s_{\mathscr{C}} 0, \alpha}\left(\mathbb{R} \times S^{1}\right), \\
w & \mapsto \phi^{2} J_{\tau} w,
\end{aligned}
$$

where the norms in the function spaces $\mathscr{b}^{k, \alpha}\left(\mathbb{R} \times S^{1}\right)$ are computed with respect to the cylindrical metric $g_{\text {cyl }}$. Observe that the Jacobi operator has been multiplied by the conformal factor $\phi^{2}$, and hence

$$
\phi^{2} J_{\tau}=\frac{1}{2}\left(\partial_{s}^{2}+\partial_{\theta}^{2}+2\left(\phi^{2}+\frac{\tau^{2}}{\phi^{2}}\right)\right) .
$$

Also, this operator depends on the parameter $\tau$. We now study the mapping properties of $\phi^{2} J_{\tau}$ as the parameter $\tau$ tends to 0 . The following result selects a range of weights for which the norm of the solution of $L_{\delta} w=f$ is controlled, uniformly as $\tau$ tends to 0 . 
Proposition 4.7. Assume that $|\delta|>1, \delta \notin \mathbb{Z}$ is fixed. Then there exist $\tau_{\delta}>0$ and $C>0$, only depending on $\delta$, such that, for all $\tau \in\left(0, \tau_{\delta}\right)$ and all $w \in e^{\delta s} \mathfrak{b}^{2, \alpha}\left(\mathbb{R} \times S^{1}\right)$, we have

$$
\left\|e^{-\delta s} w\right\|_{\mathscr{C}^{2, \alpha}\left(\mathbb{R} \times S^{1}\right)} \leq C\left\|e^{-\delta s} L_{\delta} w\right\|_{\mathscr{C}^{0, \alpha}\left(\mathbb{R} \times S^{1}\right)} .
$$

Proof. Observe that, thanks to Schauder's elliptic estimates, it is enough to prove

$$
\left\|e^{-\delta s} w\right\|_{L^{\infty}\left(\mathbb{R} \times S^{1}\right)} \leq C\left\|e^{-\delta s} \phi_{\tau}^{2} J_{\tau} w\right\|_{L^{\infty}\left(\mathbb{R} \times S^{1}\right)},
$$

provided $\tau$ is close enough to 0 . The proof of this estimate is by contradiction. Assume that, for some sequence $\tau_{n}$ tending to 0 , there exists a sequence of functions $w_{n}$ such that

$$
\left\|e^{-\delta s} w_{n}\right\|_{L^{\infty}\left(\mathbb{R} \times S^{1}\right)}=1 \quad \text { and } \quad \lim _{j \rightarrow \infty}\left\|e^{-\delta s} \phi_{\tau_{n}}^{2} J_{\tau_{n}} w_{n}\right\|_{L^{\infty}\left(\mathbb{R} \times S^{1}\right)}=0 .
$$

Pick a point $s_{n} \in \mathbb{R}$ such that $\left\|e^{-\delta s_{n}} w_{n}\left(s_{n}, \cdot\right)\right\|_{L^{\infty}\left(S^{1}\right)} \geq \frac{1}{2}$ and define the rescaled sequence

$$
\bar{w}_{n}(s, \theta):=e^{-\delta s_{n}} w_{n}\left(s+s_{n}, \theta\right) .
$$

We still have

$$
\left\|e^{-\delta s} \bar{w}_{n}\right\|_{L^{\infty}\left(\mathbb{R} \times S^{1}\right)}=1 \quad \text { and } \quad \lim _{j \rightarrow \infty}\left\|e^{-\delta s} \bar{L}_{n} \bar{w}_{n}\right\|_{L^{\infty}\left(\mathbb{R} \times S^{1}\right)}=0,
$$

where, by definition, $\bar{L}_{n}$ is defined by

$$
\bar{L}_{n}:=\partial_{s}^{2}+\partial_{\theta}^{2}+2\left(\phi_{\tau_{n}}^{2}+\frac{\tau_{n}^{2}}{\phi_{\tau_{n}}^{2}}\right)\left(\cdot+s_{n}\right) .
$$

Elliptic estimates and the Ascoli-Arzelà theorem allow us to extract some subsequence and pass to the limit as $n$ tends to $\infty$ to get a function $w_{\infty}$ which is a nontrivial solution to either

$$
\left(\partial_{s}^{2}+\partial_{\theta}^{2}\right) w_{\infty}=0
$$

or

$$
\left(\partial_{s}^{2}+\partial_{\theta}^{2}+\frac{2}{\cosh ^{2}\left(\cdot+s_{*}\right)}\right) w_{\infty}=0,
$$

according to the different cases described in Lemma 4.5. To simplify notation, we assume that $s_{*}=0$; straightforward modifications are needed to handle the general case. Observe that we also have

$$
\left\|e^{-\delta s} w_{\infty}\right\|_{L^{\infty}\left(\mathbb{R} \times S^{1}\right)} \leq 1,
$$

and $\left\|w_{\infty}(0, \cdot)\right\|_{L^{\infty}\left(S^{1}\right)} \geq \frac{1}{2}$. 
We decompose $w_{\infty}$ as

$$
w_{\infty}(s, \theta)=\sum_{j \in \mathbb{Z}} w^{(j)}(s) e^{i j \theta} .
$$

It is easy to prove that, for any solution of $(4-10), w^{(j)}$ is a linear combination of $e^{ \pm j s}$ and it is not bounded by a constant times $e^{\delta s}$ unless $\delta$ is an integer (which we have assumed not to be the case).

Similarly, if $w_{\infty}$ is a solution of (4-11), we find that $w^{(j)}$ is a solution of

$$
\left(\partial_{s}^{2}-j^{2}+\frac{2}{\cosh ^{2} s}\right) w^{(j)}=0,
$$

and is asymptotic to either $e^{j s}$ or to $e^{-j s}$ at $\pm \infty$. Inspection of the behavior of (4-12) at infinity then implies that there is no solution bounded by a constant times $e^{\delta s}$ if $|j|<|\delta|$. When $|j|>|\delta|$, inspection of the behavior of (4-12) at infinity implies that any solution is necessarily bounded, and the maximum principle then implies that this solution is identically 0 (observe that in this case $j^{2}>2$ since $|j|>|\delta|>1$, and hence the potential in (4-12) is negative).

When $j=0$, all solutions of

$$
\left(\partial_{s}^{2}+\frac{2}{\cosh ^{2} s}\right) w^{(0)}=0
$$

are linear combinations of the functions $\tanh s$ and $1-s \tanh s$ and none is bounded by a constant times $e^{\delta s}$ unless $\delta=0$ (which is not the case).

Finally — and this is the reason we had to choose $|\delta|>1$ - when $j=1$, all solutions of

$$
\left(\partial_{s}^{2}-1+\frac{2}{\cosh ^{2} s}\right) w^{(1)}=0
$$

are linear combinations of the functions $(\cosh s)^{-1}$ and $s(\cosh s)^{-1}+\sinh s$ and none is bounded by a constant times $e^{\delta s}$ unless $|\delta| \leq 1$ (which is contrary to our assumption). Again we have reached a contradiction. Having reached a contradiction in all cases, the proof of the Proposition is complete.

Thanks to the previous result, we can now describe the mapping properties of $\phi^{2} J_{\tau}$ for the range of weights $\delta$ of interest for our problem.

Proposition 4.8. Assume that $|\delta|>1, \delta \notin \mathbb{Z}$ is fixed. Then there exist $\tau_{\delta}>0$ and $C>0$, only depending on $\delta$, such that, for all $\tau \in\left(0, \tau_{\delta}\right)$, the operator $L_{\delta}$ is an isomorphism, the norm of whose inverse is bounded independently of $\tau$.

Proof. Injectivity follows at once from Proposition 4.7. As far as surjectivity is concerned, we give here a simple self-contained proof in the case where $\delta \in(1, \sqrt{2})$ (or $\delta \in(-\sqrt{2},-1)$ ). We then sketch a general proof.

To fix the ideas, assume that $\delta \in(1, \sqrt{2})$. First assume that $f \in \mathscr{C}^{0, \alpha}\left(\mathbb{R} \times S^{1}\right)$ 
has compact sets support and decompose it as

$$
f(s, \theta)=f_{0}(s)+f_{ \pm 1}(s) e^{ \pm i \theta}+\bar{f}(s, \theta),
$$

where, by definition,

$$
\bar{f}:=\sum_{j \neq 0, \pm 1} f_{j}(s) e^{i j \theta} .
$$

Observe that, if we restrict our attention to functions $\bar{w}$ whose Fourier decomposition in the $\theta$ variable is of the form

$$
\bar{w}(s, \theta)=\sum_{j \neq 0, \pm 1} w_{j}(s) e^{i j \theta}
$$

we have

$$
\int_{\mathbb{R} \times S^{1}}\left(\left|\partial_{s} \bar{w}\right|^{2}+\left|\partial_{\theta} \bar{w}\right|^{2}-2\left(\phi^{2}+\frac{\tau^{2}}{\phi^{2}}\right) \bar{w}^{2}\right) d s d \theta \geq(2-4 \tau) \int_{\mathbb{R} \times S^{1}} \bar{w}^{2} d s d \theta .
$$

This follows at once from the estimate of the potential involved in the expression of $J_{\tau}$ obtained in the proof of Proposition 4.6, namely,

$$
2\left(\phi^{2}+\frac{\tau^{2}}{\phi^{2}}\right) \leq 2+4 \tau
$$

together with the fact that

$$
\int_{\mathbb{R} \times S^{1}}\left|\partial_{\theta} \bar{w}\right|^{2} d s d \theta \geq 4 \int_{\mathbb{R} \times S^{1}} \bar{w}^{2} d s d \theta .
$$

Thus, if we assume that $\sqrt{2} \tau<1$, this inequality implies that we can solve

$$
\phi^{2} J_{\tau} \bar{w}=\bar{f}
$$

in $H^{1}\left(\mathbb{R} \times S^{1}\right)$. Elliptic estimates then imply that $\bar{w} \in \mathscr{C}^{2, \alpha}\left(\mathbb{R} \times S^{1}\right)$. Finally, the solvability of

$$
\phi^{2} J_{\tau}\left(w_{j} e^{i j \theta}\right)=f_{j} e^{i j \theta},
$$

for $j=0, \pm 1$, follows easily from integration of the associated second order ordinary differential equation starting from $-\infty$. Hence $w_{j} \equiv 0$ when $s$ is close to $-\infty$. Obviously, the function

$$
w:=w_{0}+w_{ \pm 1} e^{ \pm i \theta}+\bar{w}
$$

is a solution of the equation $\phi^{2} J_{\tau} w=f$.

We claim that, provided $\tau$ is chosen small enough, $w \in e^{\delta s} \complement^{2, \alpha}\left(\mathbb{R} \times S^{1}\right)$. Assuming that the claim is already proven, Proposition 4.7 applies and we get

$$
\left\|e^{-\delta s} w\right\|_{\mathscr{C}^{2, \alpha}\left(\mathbb{R} \times S^{1}\right)} \leq C\left\|e^{-\delta s} f\right\|_{\mathscr{C}^{0, \alpha}\left(\mathbb{R} \times S^{1}\right)}
$$


for any function $f$ having compact support. The general result, when $f$ does not necessarily have compact support, follows from a standard exhaustion argument. We choose a sequence of functions $f^{(n)} \in \mathscr{C}^{0, \alpha}\left(\mathbb{R} \times S^{1}\right)$ having compact support converging on compact sets to a given function $f \in e^{\delta s} \mathscr{C}^{0, \alpha}\left(\mathbb{R} \times S^{1}\right)$. Moreover, without loss of generality, we can assume that

$$
\left\|e^{-\delta s} f^{(n)}\right\|_{\mathscr{C}^{0, \alpha}\left(\mathbb{R} \times S^{1}\right)} \leq C\left\|e^{-\delta s} f\right\|_{\mathscr{C} 0, \alpha}\left(\mathbb{R} \times S^{1}\right)
$$

for some constant $C>0$ independent of $n \geq 0$. Thanks to the above, we have a sequence of solutions of $\phi^{2} J_{\tau} w^{(n)}=f^{(n)}$ satisfying

$$
\left\|e^{-\delta s} w^{(n)}\right\|_{\mathscr{C}^{2, \alpha}\left(\mathbb{R} \times S^{1}\right)} \leq C\left\|e^{-\delta s} f\right\|_{\mathscr{C}^{0, \alpha}\left(\mathbb{R} \times S^{1}\right)} .
$$

Extracting some subsequence and passing to the limit, one gets the existence of $w \in e^{\delta s \mathcal{C}^{0, \alpha}}\left(\mathbb{R} \times S^{1}\right)$, a solution of $\phi^{2} J_{\tau} w=f$ satisfying

$$
\left\|e^{-\delta s} w\right\|_{\mathscr{C}^{0, \alpha}\left(\mathbb{R} \times S^{1}\right)} \leq C\left\|e^{-\delta s} f\right\|_{\mathscr{C}^{0, \alpha}\left(\mathbb{R} \times S^{1}\right)} .
$$

The result then follows from Schauder's estimates.

It remains to prove the claim. We keep the notations introduced above. We first prove that $\bar{w}$ tends to 0 exponentially fast at infinity. Indeed, away from the support of $\bar{f}$, we can multiply the equation $\phi^{2} J_{\tau} \bar{w}=\bar{f}$ by $\bar{w}$ and integrate over $S^{1}$ to get

$$
\frac{1}{2} \frac{d^{2}}{d s^{2}}\left(\int_{S^{1}} \bar{w}^{2} d \theta\right)=\int_{S^{1}}\left(\left|\partial_{S} \bar{w}\right|^{2}+\left|\partial_{\theta} \bar{w}\right|^{2}-\left(\phi^{2}+\frac{\tau^{2}}{\phi^{2}}\right) \bar{w}^{2}\right) d \theta .
$$

But

$$
\int_{S^{1}}\left|\partial_{\theta} \bar{w}\right|^{2} d \theta \geq 2 \int_{S^{1}} \bar{w}^{2} d \theta
$$

and we conclude from (4-14) that

$$
\frac{d^{2}}{d s^{2}}\left(\int_{S^{1}} \bar{w}^{2} d \theta\right) \geq 4(1-2 \tau) \int_{S^{1}} \bar{w}^{2} d \theta
$$

Since we have assumed that $\delta \in(1, \sqrt{2})$, we can assume that $\tau>0$ is small enough so that

$$
\delta^{2} \leq 2(1-2 \tau)
$$

and using the fact that $\bar{w}$ is bounded, we conclude that there exists $C>0$ such that

$$
\int_{S^{1}} \bar{w}^{2} d \theta \leq C(\cosh s)^{-2 \delta}
$$

This shows that $\bar{w} \in(\cosh s)^{-\delta} L^{2}\left(\mathbb{R} \times S^{1}\right)$, and, by elliptic regularity, this implies that $\bar{w} \in(\cosh s)^{-\delta} \mathscr{C}^{2, \alpha}\left(\mathbb{R} \times S^{1}\right)$.

It remains to check that the functions $w_{0}$ and $w_{ \pm 1}$ are at most growing linearly 
at $+\infty$. This follows at once from the fact that, for $s$ large enough, these functions are solutions of the second order homogeneous ordinary differential equations

$$
\left(\partial_{s}^{2}-j^{2}+2\left(\phi^{2}+\frac{\tau^{2}}{\phi^{2}}\right)\right) w_{j}=0 .
$$

For $j=0,1$, this ordinary differential equation, whose potential is periodic, has one solution which is periodic (see the proof of Proposition 4.6), and a standard result implies that the other linearly independent solution of this ordinary differential equation is at most growing linearly (see the appendix). In particular, $w_{j} \in e^{\delta s} \complement^{2, \alpha}(\mathbb{R})$, and this completes the proof of the claim.

We briefly explain how the proof of the general result can be obtained. The idea is to solve the equation $\phi^{2} J_{\tau} \bar{w}_{s_{0}}=\bar{f}$ in $\left[-s_{0}, s_{0}\right] \times S^{1}$ with 0 boundary conditions. This can be done using the coercivity inequality (4-13). Then the proof of Proposition 4.7 can be adapted to prove that

$$
\left\|e^{-\delta s} \bar{w}_{s_{0}}\right\|_{\mathscr{C}^{2, \alpha}\left(\left[-s_{0}, s_{0}\right] \times S^{1}\right)} \leq C\left\|e^{-\delta s} \bar{f}\right\|_{\mathscr{C}^{0, \alpha}\left(\mathbb{R} \times S^{1}\right)}
$$

for some constant $C>0$ independent of $s_{0}>1$ (observe that we use the fact that the Fourier decomposition of the function $\bar{w}$ in the $\theta$ variable does not have any component over 1 and $e^{ \pm i \theta}$ ). It then remains to pass to the limit in the sequence $w_{s_{0}}$ as $s_{0}$ tends to $\infty$ to prove the existence of a solution $\bar{w}$ to $\phi^{2} J_{\tau} \bar{w}=\bar{f}$ in $\mathbb{R} \times S^{1}$, which satisfies the correct estimate.

Using similar arguments, one can give a direct proof of the following general result, which will not be needed in this paper.

Theorem 4.9. Assume that $\delta \neq \pm \delta_{j}(\tau)$ for all $j \in \mathbb{N}$. Then $L_{\delta}$ is an isomorphism.

The proof of this result follows from the general theory developed in [Pacard 2008] (see Theorem 10.2.1 on page 61 and Proposition 12.2.1 on page 81) or [Melrose 1993; Mazzeo 1991].

In what follows we restrict our attention to functions which are invariant under some symmetries. More precisely, we assume that the functions are invariant under the action on $S^{1}$ of the dihedral group $\operatorname{Dih}_{m+1}^{(2)}$ of isometries of $\mathbb{R}^{2}$ which leave a regular polygon with $m+1$ sides fixed. The operator associated to $\phi^{2} J_{\tau}$, acting on the weighted space of functions which are invariant under these symmetries, is denoted by $L_{\delta}^{\sharp}$. This time $L_{\delta}^{\sharp}$ is an isomorphism provided $\delta \neq \pm \delta_{j}$ for all $j \in \mathbb{Z}$ for which there exist eigenfunctions of $\partial_{\theta}^{2}$ which are invariant under the action of $\operatorname{Dih}_{m+1}^{(2)}$, namely, $j \notin m \mathbb{Z}$. Observe that, when $j=1$, there are no such eigenfunctions, and hence working equivariantly allows us to extend the range in which the weight parameter $\delta$ can be chosen.

Close inspection of the previous proof shows that the range in which the weight $\delta$ can be chosen so that the inverse of $L_{\delta}^{\sharp}$ remains bounded as $\tau$ tends to 0 can be 
enlarged if we work equivariantly. Even though we will not use it, we state here the corresponding result for the sake of completeness.

Proposition 4.10. Assume that $\delta \notin m \mathbb{Z}$ is fixed. Then there exist $\tau_{\delta}>0$ and $C>0$, only depending on $\delta$, such that, for all $\tau \in\left(0, \tau_{\delta}\right)$ and for all $w \in e^{\delta s} \mathscr{C}^{2, \alpha}\left(\mathbb{R} \times S^{1}\right)$, which is invariant under the action of $\mathrm{Dih}_{m+1}^{(2)}$, we have

$$
\left\|e^{-\delta s} w\right\|_{\mathscr{C}^{2, \alpha}\left(\mathbb{R} \times S^{1}\right)} \leq C\left\|e^{-\delta s} L_{\delta}^{\sharp} w\right\|_{\mathscr{C}^{0, \alpha}\left(\mathbb{R} \times S^{1}\right)} .
$$

The mean curvature of normal graphs over $\mathfrak{D}_{\tau}$. In this section, we investigate the mean curvature of a surface which is a normal graph over $\mathfrak{D}_{\tau}$. Given a smooth function $w$ (small enough) defined on $\mathfrak{D}_{\tau}$, we consider the surface parametrized by

$$
\tilde{X}(s, \theta)=X_{\tau}(s, \theta)+w(s, \theta) N_{\tau}(s, \theta) .
$$

We have the following technical result.

Lemma 4.11. The mean curvature of the surface parametrized by $\tilde{X}$ is given by

$$
H(w)=1+J_{\tau} w+\frac{1}{\phi} Q_{\tau}\left(\frac{w}{\phi}\right),
$$

where the second order differential nonlinear operator $Q_{\tau}$ depends on $\tau$ and satisfies

$$
\begin{aligned}
\| Q_{\tau}\left(v_{2}\right)- & Q_{\tau}\left(v_{1}\right) \|_{\mathscr{C}^{0, \alpha}\left([s, s+1] \times S^{1}\right)} \\
& \leq c\left(\left\|v_{1}\right\|_{\mathscr{C}^{2, \alpha}\left([s, s+1] \times S^{1}\right)}+\left\|v_{2}\right\|_{\mathscr{C}^{2, \alpha}\left([s, s+1] \times S^{1}\right)}\right)\left\|v_{2}-v_{1}\right\|_{\mathscr{C}^{2, \alpha}\left([s, s+1] \times S^{1}\right)}
\end{aligned}
$$

for some constant $c>0$ independent of $s$ and $\tau \in(0,1)$ and for all functions $v_{1}, v_{2}$ satisfying $\left\|v_{i}\right\|_{\mathscr{C}^{2, \alpha}\left([s, s+1] \times S^{1}\right)} \leq 1$.

Proof. This follows at once from Proposition 2.1 together with the fact that the functions $\phi, \tau / \phi$, and $\dot{\phi} / \phi$, as well as their derivatives, are uniformly bounded as $\tau$ tends to 0 . Indeed, we have

$$
g_{w}=g-2 w h+w^{2} k=\phi^{2}\left(\left(1-\left(\phi+\frac{\tau}{\phi}\right) \frac{w}{\phi}\right)^{2} d s^{2}+\left(1-\left(\phi-\frac{\tau}{\phi}\right) \frac{w}{\phi}\right)^{2} d \theta^{2}\right) .
$$

Hence $\phi^{-2} g_{w}$ has coefficients which are bounded functions of $w / \phi$. Similarly, the tensor $\phi^{-1}(h-w k)$ also has coefficients which are bounded functions of $w / \phi$. Using this, it is straightforward to check that the nonlinear terms in $H(w)$ are a function of $\partial_{s}^{k} \partial_{\theta}^{l} w / \phi$ for $k+l=0,1,2$ with coefficients bounded by $1 / \phi$. Finally, observe that

$$
\frac{\partial_{s} w}{\phi}=\partial_{s}\left(\frac{w}{\phi}\right)+\frac{\dot{\phi}}{\phi} \frac{w}{\phi}
$$

and hence any expressions of the form $\partial_{s} w / \phi$ can also be expressed as a linear combination (with coefficients bounded uniformly as $\tau$ tends to 0 ) of the function $w / \phi$ and its derivatives. We leave the details to the reader. 
A first fixed-point argument. We assume that we are given $\tau>0$. We define $\bar{s} \in\left(-s_{\tau}, 0\right)$ by the identity

$$
\phi_{\tau}(\bar{s})=\tau^{3 / 4}
$$

Observe that $\bar{s}$ depends on $\tau$ even though we have chosen not to make this apparent in the notation. Moreover, it follows from the proof of Proposition 4.3 that

$$
\bar{s}=\frac{1}{4} \log \tau+\mathcal{O}(1)
$$

as $\tau$ tends to 0 . We define the truncated nodoid $\mathfrak{D}_{\tau}^{+}$to be the image of $[\bar{s},+\infty) \times S^{1}$ by $X_{\tau}$. Observe that this surface has a boundary, and, thanks to Proposition 4.3, close to this boundary it can be parametrized as the vertical graph of the function

$$
x \mapsto \tau \log \frac{2|x|}{\tau}+O_{\mathscr{C} \infty}\left(\tau^{3 / 2}\right)
$$

over the annulus $\bar{D}\left(0, \tau^{3 / 4}\right)-D\left(0, \tau^{3 / 4} / 2\right)$. Moreover, $\mathfrak{D}_{\tau}^{+}$has one end in the upper half space.

In this section, we apply the implicit function theorem (or, to be more precise, a fixed-point argument for a contraction mapping) to produce an infinite-dimensional family of constant mean curvature surfaces which are close to $\mathfrak{D}_{\tau}^{+}$and have one boundary which can be described using a function $f: S^{1} \rightarrow \mathbb{R}$.

Proposition 4.12. Assume that we are given $\kappa>0$ large enough (to be fixed later on). For all $\tau>0$ small enough and for all functions $f$ invariant under the action of $\mathrm{Dih}_{m+1}^{(2)}$ satisfying (H1) (observe that (H2) is automatically satisfied) and

$$
\|f\|_{\mathscr{C}^{2, \alpha}\left(S^{1}\right)} \leq \kappa \tau^{3 / 2},
$$

there exists a constant mean curvature surface $\mathfrak{D}_{\tau, f}^{+}$with mean curvature equal to 1 that is a graph over $\mathfrak{D}_{\tau}^{+}$and has one Delaunay end asymptotic to the end of $\mathfrak{D}_{\tau}^{+}$ and one boundary. When $f=0, \mathfrak{D}_{\tau, 0}=\mathfrak{D}_{\tau}^{+}$and, close to its boundary, the surface $\mathfrak{D}_{\tau, f}$ is a vertical graph over the annulus

$$
\left\{x \in \mathbb{R}^{2}: \frac{1}{2} \tau^{3 / 4} \leq|x| \leq \tau^{3 / 4}\right\}
$$

for the function $x \mapsto U_{\tau, f}^{\uparrow}\left(\tau^{-3 / 4} x\right)$, which can be expanded as

$$
U_{\tau, f}^{\uparrow}(x)=\tau \log \frac{2}{\tau^{1 / 4}}+\tau \log |x|-W_{f}^{\mathrm{ins}}(x)+\bar{U}_{\tau, f}^{\uparrow}(x),
$$

where we recall that $W_{f}^{\mathrm{ins}}$ denotes the bounded harmonic extension of $f$ in the punctured unit disc and where

$$
\left\|\bar{U}_{\tau, 0}^{\lessgtr}\right\|_{\mathscr{C}^{2, \alpha}(\bar{D}(0,1)-D(0,1 / 2))} \leq C \tau^{3 / 2} .
$$


Moreover, the nonlinear operator

$$
\mathscr{C}^{2, \alpha}\left(S^{1}\right) \ni f \mapsto \bar{U}_{\tau, f}^{\uparrow} \in \mathscr{C}^{2, \alpha}\left(\bar{D}(0,1)-D\left(0, \frac{1}{2}\right)\right)
$$

is Lipschitz, and, given $\delta \in(-2,-1)$, we have

$$
\left\|\bar{U}_{\tau, f^{\prime}}^{\uparrow}-\bar{U}_{\tau, f}^{\nmid}\right\|_{\mathscr{C}^{2, \alpha}(\bar{D}(0,1)-D(0,1 / 2))} \leq C \tau^{(2+\delta) / 4}\left\|f^{\prime}-f\right\|_{\mathscr{C}^{2, \alpha}\left(S^{1}\right)}
$$

for some constant $C>0$, independent of $\kappa, \tau$ and $f, f^{\prime}$. Finally, $\mathfrak{D}_{\tau, f}^{+}$is invariant under the action of the dihedral group $\mathrm{Dih}_{m+1}^{(2)}$.

Before we proceed with the proof, observe that we have chosen to describe the surface near its boundary as the graph of the function $x \mapsto U_{\tau, f}^{\uparrow}\left(\tau^{-3 / 4} x\right)$, and, consequently, the function $U_{\tau, f}^{\dagger}$ is defined over the annulus $\bar{D}(0,1)-D\left(0, \frac{1}{2}\right)$. Alternatively, we could have chosen not to scale the coordinates and to have a function defined over the annulus $\bar{D}\left(0, \tau^{3 / 4}\right)-D\left(0, \tau^{3 / 4} / 2\right)$, which would be more natural. However, with this latter choice, we would have to consider, in (4-17) and (4-18), function spaces where partial derivatives are taken with respect to the vector fields $r \partial_{r}$ and $\partial_{\theta}$ to evaluate the norm of these functions, while with the former choice, the Hölder spaces are the usual ones.

Proof. The proof of this result is fairly technical but, by now, standard. To begin with, in the annular region which is the image of $(\bar{s}-2, \bar{s}+2) \times S^{1}$ by $X_{\tau}$, we modify the unit vector field $N_{\tau}$ into $\bar{N}_{\tau}$ in such a way that $\bar{N}_{\tau}$ is equal to $-e_{3}$, the downward pointing unit normal vector field on the image of $(\bar{s}-1, \bar{s}+1) \times S^{1}$ by $X_{\tau}$. Using Proposition 2.2, direct estimates imply that the expression of the mean curvature given in Lemma 4.11 has to be altered to

$$
\bar{H}(w)=1+J_{\tau} w+\frac{1}{\phi^{2}} l_{\tau} w+\frac{1}{\phi} \bar{Q}_{\tau}\left(\frac{w}{\phi}\right),
$$

where $\bar{Q}_{\tau}$ enjoys properties which are similar to the properties enjoyed by $Q_{\tau}$ and where $l_{\tau}$ is a linear second order partial differential operator in $\partial_{s}$ and $\partial_{\theta}$ whose coefficients are smooth, have support in $[\bar{s}-2, \bar{s}+2] \times S^{1}$, and are bounded (in the $\mathscr{C}^{\infty}$ topology) by a constant (independent of $\tau$ ) times $\tau^{1 / 2}$. This estimate comes from the fact that

$$
N \cdot\left(-e_{3}\right)=1+\mathcal{O}\left(\tau^{1 / 2}\right)
$$

on the image of $[\bar{s}-2, \bar{s}+2] \times S^{1}$ by $X_{\tau}$.

We assume that we are given a function $f \in \mathscr{C}^{2, \alpha}\left(S^{1}\right)$ satisfying (H1), (H2), and (4-15), and we denote by $F$ the harmonic extension of $f$ in $(\bar{s}, \infty) \times S^{1}$.

Given these data, we would like to solve the nonlinear equation

$$
\phi^{2} J_{\tau}(F+w)+l_{\tau}(F+w)+\phi \bar{Q}_{\tau}\left(\frac{F+w}{\phi}\right)=0
$$


in $(\bar{s}, \infty) \times S^{1}$. Provided $w$ is small enough and decays exponentially at infinity, this will then provide constant mean curvature surfaces which are close to a half nodoid $\mathfrak{D}_{\tau}^{+}$.

We choose an extension operator

$$
\mathscr{\mathscr { C }}_{\tau}: \mathscr{C}^{0, \alpha}\left([\bar{s}, \infty) \times S^{1}\right) \rightarrow \mathscr{C}^{0, \alpha}\left(\mathbb{R} \times S^{1}\right)
$$

such that

$$
\mathscr{E}_{\tau}(\psi)= \begin{cases}\psi & \text { in }[\bar{s}, \infty) \times S^{1} \\ 0 & \text { in }(-\infty, \bar{s}-1] \times S^{1}\end{cases}
$$

and

$$
\left\|\mathscr{E}_{\tau}(\psi)\right\|_{\mathscr{C}^{0, \alpha}\left([\bar{s}-1, \bar{s}+1] \times S^{1}\right)} \leq C\|\psi\|_{\mathscr{C}^{0, \alpha}\left([\bar{s}, \bar{s}+1] \times S^{1}\right)} .
$$

We rewrite (4-19) as

$$
\phi^{2} J_{\tau} w=-\mathscr{E}_{\tau}\left(\phi^{2} J_{\tau}(F+w)+l_{\tau} F+\phi \bar{Q}_{\tau}\left(\frac{F+w}{\phi}\right)\right),
$$

where, this time, the function $w$ is defined on all $\mathbb{R} \times S^{1}$ (to be more precise, one should say that, on the right side, we consider the restriction of $w$ to $[\bar{s}, \infty) \times S^{1}$ ).

The following estimates follow easily if one uses the fact that

$$
\frac{C}{\tau} \cosh s \leq \phi \leq C \tau \cosh s \quad \text { in }\left(-s_{\tau}, s_{\tau}\right)
$$

for some $C>1$, and also that $\phi$ is periodic of period $2 s_{\tau}$. Assume that $\delta \in(-2,-1)$ is fixed. It is easy to check that there exists a constant $c>0$ (independent of $\kappa$ ) and a constant $c_{\kappa}>0$ (depending on $\kappa$ ) such that

$$
\begin{aligned}
\left\|e^{-\delta \mathscr{\mathscr { C }}_{\tau}}\left(\left(\phi^{2}+\frac{\tau^{2}}{\phi^{2}}\right) F\right)\right\|_{\mathscr{C}^{0, \alpha}\left(\mathbb{R} \times S^{1}\right)} & \leq c \tau^{1 / 2}\|f\|_{\mathscr{C}^{2, \alpha}\left(S^{1}\right)}, \\
\left\|e^{-\delta s_{\mathscr{C}_{\tau}}}\left(l_{\tau}(F+w)\right)\right\|_{\mathscr{C}^{0, \alpha}\left(\mathbb{R} \times S^{1}\right)} & \leq c \tau^{1 / 2}\left(\left\|e^{-\delta s} w\right\|_{\mathscr{C}_{\delta}^{2, \alpha}\left(\mathbb{R} \times S^{1}\right)}+\tau^{-\delta / 4}\|f\|_{\mathscr{C}^{2, \alpha}\left(S^{1}\right)}\right),
\end{aligned}
$$

and we also have

$$
\begin{aligned}
\left\|e^{-\delta s \mathscr{E}_{\tau}}\left(\phi Q_{\tau}\left(\frac{w^{\prime}+F^{\prime}}{\phi}\right)-\phi Q_{\tau}\left(\frac{w+F}{\phi}\right)\right)\right\|_{\mathscr{C}^{0, \alpha}\left(\mathbb{R} \times S^{1}\right)} \\
\quad \leq c_{\kappa}\left(\tau^{3 / 4}\left\|e^{-\delta s}\left(w^{\prime}-w\right)\right\|_{\mathscr{C}_{\delta}^{2, \alpha}\left(\mathbb{R} \times S^{1}\right)}+\tau^{(3-\delta) / 4}\left\|f^{\prime}-f\right\|_{\mathscr{C}^{2, \alpha}\left(S^{1}\right)}\right),
\end{aligned}
$$

provided $w$ and $w^{\prime}$ satisfy

$$
\left\|e^{-\delta s} w\right\|_{\mathscr{C}^{2, \alpha}\left(\mathbb{R} \times S^{1}\right)}+\left\|e^{-\delta s} w^{\prime}\right\|_{\mathscr{C}^{2, \alpha}\left(\mathbb{R} \times S^{1}\right)} \leq C_{\kappa} \tau^{2}
$$

for some fixed constant $C_{\kappa}>0$. Here $F$ and $F^{\prime}$ are the harmonic extensions of the boundary data $f$ and $f^{\prime}$, respectively.

At this stage, we make use of Proposition 4.8 (or, more precisely, its equivariant version) to rephrase (4-20) as a fixed-point problem in $e^{\delta s} \mathfrak{b}^{2, \alpha}\left(\mathbb{R} \times S^{1}\right)$. The 
estimates we have just derived are precisely enough to solve this nonlinear problem using a fixed-point argument for contraction mappings in the ball of radius $C_{\kappa} \tau^{2}$ in $e^{\delta s} \mathscr{C}^{2, \alpha}\left(\mathbb{R} \times S^{1}\right)$, where $C_{\kappa}$ is a constant which is fixed large enough. Therefore, for all $\tau>0$ small enough, we find a solution $w$ of (4-20) satisfying

$$
\left\|e^{-\delta s} w\right\|_{\mathscr{C}^{2, \alpha}\left(\mathbb{R} \times S^{1}\right)} \leq C_{\kappa} \tau^{2} .
$$

In addition, it follows from the above estimates that

$$
\left\|e^{-\delta s}\left(w^{\prime}-w\right)\right\|_{\mathscr{C}^{2, \alpha}\left(\mathbb{R} \times S^{1}\right)} \leq C_{\kappa} \tau^{1 / 2}\left\|f^{\prime}-f\right\|_{\mathscr{C}^{2, \alpha}\left(S^{1}\right)},
$$

where $w$ (respectively $w^{\prime}$ ) is the solution associated to $f$ (respectively $f^{\prime}$ ).

To complete the result, it is enough to change coordinates $r=\phi(s)$ in the range where $\frac{1}{2} \tau^{3 / 4} \leq r \leq 2 \tau^{3 / 4}$ and $|s-\bar{s}| \leq 1$. There is no real difficulty in deriving the estimates (4-17) and (4-18), which follow from Proposition 4.7 and the estimate for $w$. There is a subtlety here: if we change variables $r=\phi(s)$ for $\frac{1}{2} \tau^{3 / 4} \leq r \leq 2 \tau^{3 / 4}$ and $|s-\bar{s}| \leq 1$, then $F(s)$ is not equal to $W_{f}^{\text {ins }}(\phi(s))$, because $s$ does not correspond to the cylindrical coordinate $r=e^{t}$ in $\mathbb{R}^{2}-\{0\}$ ! In fact, we have

$$
F(s)=W_{f}^{\text {ins }}\left(e^{\bar{s}-s}\right),
$$

and $\tau^{-3 / 4} r=\phi(s) / \phi(\bar{s})$ and is not equal to $e^{\bar{s}-s}$. Nevertheless, using the expansion of $\phi$ we have derived, we easily check that

$$
\|F(\bar{s}-\log \phi(s)+\log \phi(\bar{s}))-F(s)\|_{\mathscr{C}^{2, \alpha}\left([\bar{s}, \bar{s}+2] \times S^{1}\right)} \leq c \tau^{1 / 2}\|f\|_{\mathscr{C}^{2, \alpha}\left(S^{1}\right)}
$$

for some constant $c>0$ independent of $\tau$.

\section{The catenoid}

Parametrization and notations. We recall some well-known facts about catenoids in Euclidean space. The normalized catenoid $\mathfrak{C}$ is the minimal surface of revolution, parametrized by

$$
Y_{0}(s, \theta):=(\cosh s \cos \theta, \cosh s \sin \theta, s),
$$

where $(s, \theta) \in \mathbb{R} \times S^{1}$. The induced metric on $\mathfrak{C}$ is given by

$$
g_{0}:=(\cosh s)^{2}\left(d s^{2}+d \theta^{2}\right),
$$

and it is easy to check that the second fundamental form is given by

$$
h_{0}:=d s^{2}-d \theta^{2}
$$

when the unit normal vector field is chosen to be

$$
N_{0}:=\frac{1}{\cosh s}(\cos \theta, \sin \theta,-\sinh s) .
$$


In particular, the formulae for the induced metric and the second fundamental form imply that the mean curvature of the surface $\mathfrak{C}$ is constant and equal to 0 .

In the above defined coordinates, the Jacobi operator about the catenoid is given by

$$
J_{0}:=\frac{1}{2 \cosh ^{2} s}\left(\partial_{s}^{2}+\partial_{\theta}^{2}+\frac{2}{\cosh ^{2} s}\right) .
$$

Refined asymptotics. We are interested in the parametrization of the catenoid as a (multivalued) vertical graph over the horizontal plane. We consider, for example, the lower part of the catenoid as the graph over the complement of the unit disc in the horizontal plane for the function $u_{0}$. Namely, $u_{0}$ is the negative function defined by

$$
u_{0}(\cosh s)=s
$$

for all $s \leq 0$. It is easy to check that

$$
s=-\log (2 r)+\mathfrak{O}_{\dot{\varphi} \infty}\left(r^{-2}\right),
$$

and hence the lower end of the catenoid can also be parametrized as a vertical graph over $\mathbb{R}^{2}-D(0,1)$ by

$$
(r, \theta) \mapsto\left(r \cos \theta, r \sin \theta, u_{0}(r)\right) .
$$

With little work, one proves the following.

Lemma 5.1. The expansion

$$
u_{0}(r)=-\log (2 r)+0_{\mathscr{C} \infty}\left(r^{-2}\right)
$$

holds in $\mathbb{R}^{2}-D(0,2)$.

Mapping properties of the Jacobi operator about the catenoid. The functional analysis of the Jacobi operator about the catenoid is well understood, and some results can be found, for example, in [Mazzeo et al. 2001]. Again the indicial roots of $J_{0}$ characterize the asymptotic behavior of the solutions of the homogeneous problem $J_{0, j} w=0$, where

$$
J_{0, j}:=\frac{1}{2 \cosh ^{2} s}\left(\partial_{s}^{2}-j^{2}+\frac{2}{\cosh ^{2} s}\right) .
$$

It is easy to see that the indicial roots of $J_{0, j}$ are equal to $\pm j$, and hence the indicial roots of $J_{0}$ are equal to $\pm j$ for $j \in \mathbb{N}$.

For all $\delta \in \mathbb{R}$, we define the operator

$$
\begin{aligned}
\mathscr{L}_{\delta}:(\cosh s)^{\delta} \mathscr{C}^{2, \alpha}\left(\mathbb{R} \times S^{1}\right) & \rightarrow(\cosh s)^{\delta} \mathscr{C}^{0, \alpha}\left(\mathbb{R} \times S^{1}\right), \\
w & \mapsto(\cosh s)^{2} J_{0} w,
\end{aligned}
$$


where, as usual, the norms in the function spaces $\mathscr{C}^{k, \alpha}\left(\mathbb{R} \times S^{1}\right)$ are computed with respect to the cylindrical metric $g_{\text {cyl }}$.

Paralleling what we have proven in Section 4, we have the following.

Proposition 5.2. Assume that $\delta \in(-2,2)$. Then there exists $C>0$, only depending on $\delta$, such that, for all $\bar{w} \in(\cosh s)^{\delta} \mathscr{C}^{2, \alpha}\left(\mathbb{R} \times S^{1}\right)$, we have

$$
\left\|(\cosh s)^{-\delta} \bar{w}\right\|_{\mathscr{C}^{2, \alpha}\left(\mathbb{R} \times S^{1}\right)} \leq C\left\|(\cosh s)^{-\delta} \mathscr{L}_{\delta} \bar{w}\right\|_{\mathscr{C}^{0, \alpha}\left(\mathbb{R} \times S^{1}\right)},
$$

provided

$$
\int_{S^{1}} \bar{w}(s, \theta) d \theta=\int_{S^{1}} \bar{w}(s, \theta) e^{ \pm i \theta} d \theta=0
$$

for all $s \in \mathbb{R}$.

Proof. The proof is parallel to that of Proposition 4.7 and is left to the reader.

The following result follows from the general theory developed in [Pacard 2008] (see Theorem 10.2.1 on page 61 and Proposition 12.2.1 on page 81) or [Lockhart and McOwen 1985; Melrose 1993; Mazzeo 1991]. For the sake of completeness we provide a self-contained proof.

Theorem 5.3. Assume $\delta \in(1,2)$. Then $\mathscr{L}_{\delta}$ is surjective and has a 6-dimensional kernel.

Proof. The proof is similar to that of Proposition 4.8. Recall that the action of rigid motions and dilations provides many Jacobi fields. For example,

$$
J_{0}(\tanh s)=0 \quad \text { and } \quad J_{0}(1-s \tanh s)=0,
$$

which either follow from direct computation or from the fact that these are the Jacobi fields associated to the group of vertical translations and the group of dilations centered at the origin.

Similarly

$$
J_{0}\left(\frac{1}{\cosh s} e^{ \pm i \theta}\right)=0 \quad \text { and } \quad J_{0}\left(\left(\sinh s+\frac{1}{\cosh s}\right) e^{ \pm i \theta}\right)=0,
$$

which again either follows from direct computation or from the fact that these are the Jacobi fields associated to the group of horizontal translations and the group of rotations about the vertical axis, centered at the origin.

This already shows that when $\delta \in(1,2)$ the kernel of $\mathscr{L}_{\delta}$ is at least 6-dimensional.

We first assume that $f \in \mathscr{C}^{0, \alpha}\left(\mathbb{R} \times S^{1}\right)$ has compact support and we decompose it as $f(s, \theta)=f_{0}(s)+f_{ \pm 1}(s) e^{ \pm i \theta}+\bar{f}(s, \theta)$, where, by definition,

$$
\bar{f}:=\sum_{j \neq 0, \pm 1} f_{j}(s) e^{i j \theta} .
$$


If we restrict our attention to functions $\bar{w}$ whose Fourier decomposition in the $\theta$ variable is of the form

$$
\bar{w}(s, \theta)=\sum_{j \neq 0, \pm 1} w_{j}(s) e^{i j \theta}
$$

we have

$$
\int_{\mathbb{R} \times S^{1}}\left(\left|\partial_{s} \bar{w}\right|^{2}+\left|\partial_{\theta} \bar{w}\right|^{2}-\frac{2}{\cosh ^{2} s} \bar{w}^{2}\right) d s d \theta \geq 2 \int_{\mathbb{R} \times S^{1}} \bar{w}^{2} d s d \theta .
$$

Therefore, we can solve

$$
(\cosh s)^{2} J_{0} \bar{w}=\bar{f}
$$

in $H^{1}\left(\mathbb{R} \times S^{1}\right)$. Elliptic estimates then imply that $\bar{w} \in \mathscr{C}^{2, \alpha}\left(\mathbb{R} \times S^{1}\right)$.

Obviously $\bar{w} \in(\cosh s)^{\delta} \mathscr{C}^{2, \alpha}\left(\mathbb{R} \times S^{1}\right)$, since $\delta>0$. Proposition 5.2 applies, and we get

$$
\left\|(\cosh s)^{-\delta} \bar{w}\right\|_{\mathscr{C}^{2, \alpha}\left(\mathbb{R} \times S^{1}\right)} \leq C\left\|(\cosh s)^{-\delta} f\right\|_{\mathscr{C}^{0, \alpha}\left(\mathbb{R} \times S^{1}\right)}
$$

for any function $f$ having compact support. The general result, when $f$ does not necessarily have compact support, follows from a standard exhaustion argument.

Finally, the solvability of

$$
(\cosh s)^{2} J_{0}\left(w_{j} e^{i j \theta}\right)=f_{j} e^{i j \theta}
$$

for $j=0, \pm 1$, follows easily from integration of the associated second order ordinary differential equation starting from 0 (with initial data and initial velocity equal to $0)$. We have, explicitly,

$$
w_{j}=A_{j}^{+} \int_{0}^{s} A_{j}^{-}(t) f_{j}(t) d t-A_{j}^{-} \int_{0}^{s} A_{j}^{+}(t) f_{j}(t) d t,
$$

where $A_{j}^{ \pm}$are the two independent solutions of

$$
\left(\partial_{s}^{2}-j^{2}+\frac{2}{\cosh ^{2} s}\right) A_{j}^{ \pm}=0,
$$

which are given in (5-2) and (5-3) and are normalized so that their Wronskian is equal to 1 . Direct estimates imply that

$$
\left\|(\cosh s)^{-\delta} w_{j}\right\|_{\mathscr{C}^{2, \alpha}(\mathbb{R})} \leq C\left\|(\cosh s)^{-\delta} f\right\|_{\mathscr{C}^{0, \alpha}\left(\mathbb{R} \times S^{1}\right)},
$$

provided $\delta>1$ (more precisely, $\delta>0$ is needed to derive the estimate for $w_{0}$ and $\delta>1$ is needed to derive the estimate for $w_{ \pm 1}$ ). We set $w=w_{0}+w_{ \pm 1} e^{ \pm i \theta}+\bar{w}$. This completes the proof of the fact that the operator $\mathscr{L}_{\delta}$ is surjective when $\delta \in(1,2)$. The fact that this operator, restricted to the space of functions satisfying the orthogonality conditions (5-1), is injective follows from Proposition 5.2. Hence the kernel of $\mathscr{L}_{\delta}$ is 6-dimensional. 
The mean curvature of normal graphs over the catenoid. We consider in this section the mean curvature of a surface which is a normal graph over $\mathfrak{C}$. Hence, for some smooth (small enough) function $w$ defined on $\mathfrak{C}$, we consider the surface parametrized by

$$
Y(s, \theta)=Y_{0}(s, \theta)+w(s, \theta) N_{0}(s, \theta) .
$$

We have the following technical result.

Lemma 5.4. The mean curvature of the surface parametrized by $Y$ is given by

$$
H(w)=J_{0} w+\frac{1}{\cosh s} Q_{0}\left(\frac{w}{\cosh s}\right),
$$

where the nonlinear second order differential operator $Q_{0}$ satisfies

$$
\begin{aligned}
\| Q_{0}\left(v_{2}\right)- & Q_{0}\left(v_{1}\right) \|_{\mathscr{C}^{0, \alpha}\left([s, s+1] \times S^{1}\right)} \\
& \leq c\left(\left\|v_{1}\right\|_{\mathscr{C}^{2, \alpha}\left([s, s+1] \times S^{1}\right)}+\left\|v_{2}\right\|_{\mathscr{C}^{2, \alpha}\left([s, s+1] \times S^{1}\right)}\right)\left\|v_{2}-v_{1}\right\|_{\mathscr{C}^{2, \alpha}\left([s, s+1] \times S^{1}\right)}
\end{aligned}
$$

for some constant $c>0$ independent of $s$ and $\tau \in(0,1)$ and for all functions $v_{1}, v_{2}$ satisfying $\left\|v_{i}\right\|_{\mathscr{C}^{2, \alpha}\left([s, s+1] \times S^{1}\right)} \leq 1$.

Proof. This result is already proven in [Mazzeo and Pacard 2001]. In any case, a simple proof follows easily from Proposition 2.1 together with the fact that

$$
g_{w}=\cosh ^{2} s\left(\left(1-\frac{w}{\cosh ^{2} s}\right)^{2} d s^{2}+\left(1+\frac{w}{\cosh ^{2} s}\right)^{2} d \theta^{2}\right) .
$$

We leave the details to the reader.

A second fixed-point argument. Assume that $\tau, \tilde{\tau}>0$ are chosen small enough and satisfy

$$
\left|\tilde{\tau}-\frac{\tau}{m+1}\right| \leq \kappa \tau^{3 / 2},
$$

where the constant $\kappa>0$ is large enough; its value will be fixed in Section 7. The rationale for this estimate is also explained in Section 7 . We define $\tilde{s}>0$ by

$$
\tilde{\tau} \cosh \tilde{s}=\tau^{3 / 4} .
$$

Observe that $\tilde{s}$ depends on both $\tau$ and $\tilde{\tau}$ even though we have chosen not to make this apparent in the notation. It is easy to check that $\tilde{s}=-(1 / 4) \log \tau+\mathcal{O}(1)$. We define the truncated catenoid $\mathfrak{C}_{\tilde{\tau}}$ to be the image of $[-\tilde{s}, \tilde{s}] \times S^{1}$ by $\tilde{\tau} Y_{0}$ (to simplify the notations, we do not write the dependence of this surface on the parameter $\tau$ ).

Building on the previous analysis, we prove the existence of constant mean curvature surfaces which are close to the truncated catenoid $\mathfrak{C}_{\tilde{\tau}}$ and have two boundaries which can be described by some function $f: S^{1} \rightarrow \mathbb{R}$. We also require 
that the surfaces are invariant under the action of the symmetry with respect to the horizontal plane. More precisely, we have the following.

Proposition 5.5. Assume we are given $\kappa>0$ large enough (to be fixed later). For all $\tau, \tilde{\tau}>0$ small enough satisfying (5-5) and for all functions $f$ invariant under the action of the $\mathrm{Dih}_{m+1}^{(2)}$ satisfying (H1) (notice that $(\mathrm{H} 2)$ is automatically satisfied) and

$$
\|f\|_{\mathscr{C}^{2, \alpha}\left(S^{1}\right)} \leq \kappa \tau^{3 / 2},
$$

there exists a constant mean curvature 1 surface $\mathfrak{C}_{\tilde{\tau}, f}$ which is close to $\mathfrak{C}_{\tilde{\tau}}$ and has two boundaries. The surface $\mathfrak{C}_{\tilde{\tau}, f}$ is invariant under the action of $\mathscr{S}_{3}$, the symmetry with respect to the horizontal plane $x_{3}=0, \mathscr{Y}_{2}$, the symmetry with respect to the plane $x_{2}=0$. And, close to its lower boundary, the surface $\mathfrak{C}_{\tilde{\tau}, f}$ is a vertical graph over the annulus

$$
\left\{x \in \mathbb{R}^{2}: \frac{1}{2} \tau^{3 / 4} \leq|x| \leq \tau^{3 / 4}\right\},
$$

for some function $x \mapsto U_{\tilde{\tau}, f}^{\downarrow}\left(\tau^{-3 / 4} x\right)$ which can be expanded as

$$
U_{\tilde{\tau}, f}^{\downarrow}(x)=-\tilde{\tau} \log \frac{2 \tau^{3 / 4}}{\tilde{\tau}}-\tilde{\tau} \log |x|+W_{f}^{\text {ins }}(x)+\bar{U}_{\tilde{\tau}, f}^{\downarrow}(x),
$$

where we recall that $W_{f}^{\mathrm{ins}}$ denotes the bounded harmonic extension of $f$ in the punctured unit disc and where

$$
\left.\left\|\bar{U}_{\tilde{\tau}, 0}^{\downarrow}\right\|_{\mathscr{C}^{2, \alpha}(\bar{D}(0,1)-D}\left(0, \frac{1}{2}\right)\right) \leq C \tau^{3 / 2} .
$$

Moreover, the nonlinear mapping

$$
\mathscr{C}^{2, \alpha}\left(S^{1}\right) \ni f \mapsto \bar{U}_{\tilde{\tau}, f}^{\downarrow} \in \mathscr{C}^{2, \alpha}\left(\bar{D}(0,1)-D\left(0, \frac{1}{2}\right)\right)
$$

is Lipschitz, and, given $\delta \in(1,2)$, we have

$$
\left\|\bar{U}_{\tilde{\tau}, f^{\prime}}^{b}-\bar{U}_{\tilde{\tau}, f}^{b}\right\|_{\mathscr{C}^{2, \alpha}\left(\bar{D}(0,1)-D\left(0, \frac{1}{2}\right)\right)} \leq C \tau^{(2-\delta) / 4}\left\|f^{\prime}-f\right\|_{\mathscr{C}^{2, \alpha}\left(S^{1}\right)}
$$

for some constant $C>0$ independent of $\kappa, \tau, \tilde{\tau}$ and $f, f^{\prime}$. The function $\bar{U}_{\tilde{\tau}, f}^{b}$ depends continuously on $\tilde{\tau}$.

Proof. The proof of this result is very similar to the proof of Proposition 4.12, so we cover only the main differences.

Again, in the annular region which is the image of $(-\tilde{s}-2,-\tilde{s}+2) \times S^{1}$ by $Y_{0}$, we modify the unit vector field $N_{0}$ into $\bar{N}_{0}$ in such a way that $\bar{N}_{0}$ is equal to $e_{3}$ on the image of $(-\tilde{s}-1, \tilde{s}+1) \times S^{1}$ by $\tilde{\tau} Y_{0}$. We perform a similar modification on the upper half of the catenoid, on the image of $(\tilde{s}-2, \tilde{s}+2) \times S^{1}$ by $Y_{0}$, so that our construction remains invariant under the action of the symmetry with respect 
to the horizontal plane. In this case, using Proposition 2.2, one can check that the expression of the mean curvature given in Lemma 4.11 has to be altered to

$$
\bar{H}(w)=J_{0} w+\frac{1}{\cosh ^{2} s} l_{0} w+\frac{1}{\cosh s} \bar{Q}_{0}\left(\frac{w}{\cosh s}\right),
$$

where $\bar{Q}_{0}$ enjoys properties which are similar to the properties enjoyed by $Q_{0}$ and where $l_{0}$ is a linear second order partial differential operator in $\partial_{s}$ and $\partial_{\theta}$ whose coefficients are smooth, supported in $(-\tilde{s}-2,-\tilde{s}+2) \times S^{1}$ and in $(\tilde{s}-2, \tilde{s}+2) \times S^{1}$, and are bounded (in the smooth topology) by a constant, independent of $\tau$, times $\tau^{1 / 2}$

We assume that $f$ is chosen to satisfy (H1), (H2), and

$$
\|f\|_{\mathscr{C}^{2, \alpha}\left(S^{1}\right)} \leq \kappa \tau^{1 / 2} .
$$

Observe that the norm of the boundary data $f$ is bounded by a constant times $\tau^{1 / 2}$ and not $\tau^{3 / 2}$, the reason being that we are going to perturb the image of $[-\tilde{s}, \tilde{s}] \times S^{1}$ by $Y_{0}$ and then scale the surface we obtain by a factor $\tilde{\tau}$ instead of perturbing $\mathfrak{C}_{\tilde{\tau}}$, which is the image of $[-\tilde{s}, \tilde{s}] \times S^{1}$ by $\tilde{Y}_{0}$. This is also the reason the equation we solve is $\bar{H}(w)=\tilde{\tau}$ and not $\bar{H}(w)=1$.

We denote by $F$ the harmonic extension of $f$ in $(-\infty, \tilde{s}) \times S^{1}$ and we set

$$
\tilde{F}(s, \theta):=F(s, \theta)+F(-s, \theta),
$$

which is well defined in $[-\tilde{s}, \tilde{s}] \times S^{1}$. One should be aware that the boundary data of $F$ is not exactly equal to $f$, but the difference between $F$ and $f$ on the boundary tends to 0 as $\tau$ tends to 0 . More precisely, we have

$$
\|F-\tilde{F}\|_{\mathscr{C}^{2, \alpha}\left([\tilde{s}-1, \tilde{s}] \times S^{1}\right)} \leq C \tau\|f\|_{\mathscr{C}^{2, \alpha}\left(S^{1}\right)} .
$$

We would like to solve the equation

$$
(\cosh s)^{2} J_{0}(\tilde{F}+w)+l_{0}(\tilde{F}+w)+\cosh s Q_{0}\left(\frac{\tilde{F}+w}{\cosh s}\right)=(\cosh s)^{2} \tilde{\tau}
$$

in $(-\tilde{s}, \tilde{s}) \times S^{1}$. This will provide constant mean curvature surfaces with mean curvature equal to $\tilde{\tau}$ which are close to the truncated catenoid. Again, the solvability of this nonlinear problem follows from a fixed-point theorem for a contraction mapping.

We choose

$$
\overline{\mathscr{E}}_{\tau}: \mathscr{C}^{0, \alpha}\left([-\tilde{s}, \tilde{s}] \times S^{1}\right) \rightarrow \mathscr{C}^{0, \alpha}\left(\mathbb{R} \times S^{1}\right),
$$

an extension operator, such that

$$
\begin{cases}\overline{\mathscr{E}}_{\tau}(\psi)=\psi & \text { in }[-\tilde{s}, \tilde{s}] \times S^{1}, \\ \overline{\mathscr{E}}_{\tau}(\psi)=0 & \text { in }((-\infty,-\tilde{s}-1] \cup[\tilde{s}+1, \infty)) \times S^{1},\end{cases}
$$


and

$$
\begin{aligned}
\left\|\overline{\mathscr{E}}_{\tau}(\psi)\right\|_{\mathscr{C}^{0, \alpha}\left([-\tilde{s}-1,-\tilde{s}+1] \times S^{1}\right)} & \leq C\|\psi\|_{\mathscr{C}^{0, \alpha}\left([-\tilde{s},-\tilde{s}+1] \times S^{1}\right)}, \\
\left\|\overline{\mathscr{E}}_{\tau}(\psi)\right\|_{\mathscr{C}^{0, \alpha}\left([\tilde{s}-1, \tilde{s}+1] \times S^{1}\right)} & \leq C\|\psi\|_{\mathscr{C}^{0, \alpha}\left(\left[\tilde{s}-1, \bar{s}^{\prime}\right] \times S^{1}\right)} .
\end{aligned}
$$

We rewrite (5-10) as

$(5-11)(\cosh s)^{2} J_{0} w=\overline{\mathscr{C}}_{\tau}\left((\cosh s)^{2}\left(\tilde{\tau}-J_{0} \tilde{F}\right)-l_{0}(\tilde{F}+w)-\cosh s Q_{0}\left(\frac{\tilde{F}+w}{\cosh s}\right)\right)$.

Again, on the right side it is understood that we consider the image by $\overline{\mathscr{E}}_{\tau}$ of the restriction of the functions to $[-\tilde{s}, \tilde{s}] \times S^{1}$.

We assume that $\delta \in(1,2)$ is fixed. It is easy to check that there exists a constant $c>0$ (independent of $\kappa$ ) and a constant $c_{\kappa}>0$ (depending on $\kappa$ ) such that

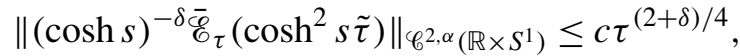

$$
\begin{aligned}
& \left\|(\cosh s)^{-\delta \overline{\mathscr{E}}_{\tau}}\left(\frac{\tilde{F}}{\cosh ^{2} S}\right)\right\|_{\mathscr{C}^{2, \alpha}\left(\mathbb{R} \times S^{1}\right)} \leq c \tau^{1 / 2}\|f\|_{\mathscr{C}^{2, \alpha}\left(S^{1}\right)},
\end{aligned}
$$

$\left\|(\cosh s)^{-\delta \overline{\mathscr{C}}_{\tau}}\left(l_{0}(\tilde{F}+w)\right)\right\|_{\mathscr{C}^{2, \alpha}\left(\mathbb{R} \times S^{1}\right)}$

$$
\leq c \tau^{1 / 2}\left(\|f\|_{\mathscr{C}^{2, \alpha}\left(S^{1}\right)}+\tau^{\delta / 4}\left\|(\cosh s)^{-\delta} w\right\|_{\mathscr{C}^{2, \alpha}\left(\mathbb{R} \times S^{1}\right)},\right),
$$

and

$$
\begin{aligned}
\left\|(\cosh s)^{-\delta \overline{\mathscr{C}}_{\tau}}\left(\cosh s Q\left(\frac{w^{\prime}+\tilde{F}^{\prime}}{\cosh s}\right)-\cosh s Q\left(\frac{w+\tilde{F}}{\cosh s}\right)\right)\right\|_{\mathscr{C}^{2, \alpha}\left(\mathbb{R} \times S^{1}\right)} \\
\leq c_{\kappa}\left(\tau^{3 / 4}\left\|(\cosh s)^{-\delta}\left(w^{\prime}-w\right)\right\|_{\mathscr{C}^{2, \alpha}\left(\mathbb{R} \times S^{1}\right)}+\tau^{(1+\delta) / 4}\left\|f^{\prime}-f\right\|_{\mathscr{C}^{2, \alpha}\left(S^{1}\right)}\right),
\end{aligned}
$$

provided $w$ and $w^{\prime}$ satisfy

$$
\left\|(\cosh s)^{-\delta} w\right\|_{\mathscr{C}^{2, \alpha}\left(\mathbb{R} \times S^{1}\right)}+\left\|(\cosh s)^{-\delta} w^{\prime}\right\|_{\mathscr{C}^{2, \alpha}\left(\mathbb{R} \times S^{1}\right)} \leq C \tau^{(2+\delta) / 4}
$$

for some fixed constant $C>0$ (independent of $\kappa, \tau$, and $f$ ). Here $\tilde{F}$ and $\tilde{F}^{\prime}$ are the harmonic extensions of the boundary data $f$ and $f^{\prime}$, respectively.

Now we make use of Theorem 5.3 to rephrase the problem as a fixed-point problem, and the previous estimates are precisely enough to solve this nonlinear problem using a fixed-point argument for contraction mappings in the ball of radius $C \tau^{(2+\delta) / 4}$ in $(\cosh s)^{\delta} \mathscr{C}^{2, \alpha}\left(\mathbb{R} \times S^{1}\right)$, where $C>0$ is fixed large enough independent of $\kappa$, provided $\tau$ is small enough. Then, for all $\tau>0$ small enough, we find that there exists constants $C>0$ (independent of $\kappa$ ) and $C_{\kappa}>0$ (depending on $\kappa$ ) such that, for all functions $f$ satisfying (H1), (H2), and (4-15), there exists a unique solution $w$ of (4-20) satisfying

$$
\left\|(\cosh s)^{\delta} w\right\|_{\mathscr{C}^{2, \alpha}\left(\mathbb{R} \times S^{1}\right)} \leq C \tau^{(2+\delta) / 4} .
$$


In addition, we have the estimate

$$
\left\|e^{-\delta s}\left(w^{\prime}-w\right)\right\|_{\mathscr{C}^{2, \alpha}\left(\mathbb{R} \times S^{1}\right)} \leq C_{\kappa} \tau^{1 / 2}\left\|f^{\prime}-f\right\|_{\mathscr{C}^{2, \alpha}\left(S^{1}\right)},
$$

where $w$ (respectively $w^{\prime}$ ) is the solution associated to $f$ (respectively $f^{\prime}$ ).

To complete the result, we simply shrink the surface we have obtained by a factor $\tilde{\tau}$ to get a surface whose mean curvature is constant and equal to 1 . The description of this surface close to its boundary follows from the arguments already used in the proof of Proposition 4.12. Observe that the solution of (4-20) is obtained through a fixed-point theorem for contraction mappings, and it is classical to check that the solution we obtain depends continuously on the parameters of the construction. In particular, the constant mean curvature surface we obtain depends continuously on $\tilde{\tau}$ (in fact one can also prove that the surface depends smoothly on $\tilde{\tau}$, but we shall not use this property).

To prove that, near its lower boundary, the surface we have obtained is a vertical graph for some function which enjoys the decomposition (5-7), we make use of the expansion in Lemma 5.1, and we follow the steps of the construction. Notice that $\bar{U}_{\tilde{\tau}, f}^{l}$ collects many remainders: the one coming from the expansion in Lemma 5.1, the difference between $F$ and $\tilde{F}$, the solution $w$ of the fixed-point problem. Also, the change of coordinates which takes into account that the variable $s$ does not correspond to the cylindrical coordinates in $\mathbb{R}^{2}-\{0\}$.

\section{The unit sphere}

Notations and definitions. We denote by $x_{1}, x_{1}, x_{3}$ the coordinates in $\mathbb{R}^{3}$, and by $\mathscr{S}_{j}$ the symmetry with respect to the plane $x_{j}=0$. For all $m \in \mathbb{N}$, we write $\mathscr{R}_{m+1}$ for a rotation of angle $2 \pi /(m+1)$ about the $x_{3}$-axis. With slight abuse of notation, we will keep the same notation to denote the restriction of these isometries to the horizontal plane.

We define $z_{0}, \ldots, z_{m} \in S^{1}$ to be vertices of a regular polygon with $m+1$ edges in the plane. Without loss of generality, we can choose

$$
z_{0}:=(1,0)=e_{1} \in \mathbb{R}^{2}
$$

and, for $j=1, \ldots, m-1, z_{j+1} \in \mathbb{R}^{2}$ is the image of $z_{j}$ by $\mathscr{R}_{m+1}$. Thus, if we identify the horizontal plane with $\mathbb{C}$, the vertices of the polygon are exactly the $(m+1)$-th roots of unity. Recall that the dihedral group of symmetries of $\mathbb{R}^{2}$ leaving this polygon fixed has been denoted by $\operatorname{Dih}_{m+1}^{(2)}$. It is generated by $\mathscr{R}_{m+1}$ and $\mathscr{Y}_{2}$.

Let $S^{2}$ be the unit sphere in $\mathbb{R}^{3}$. The upper half hemisphere of $S^{2}$ can be parametrized by

$$
X^{\dagger}(x):=\left(x, \sqrt{1-|x|^{2}}\right),
$$


while the lower hemisphere is parametrized by

$$
X^{\downarrow}(x):=\left(x,-\sqrt{1-|x|^{2}}\right),
$$

where, in both cases, $x \in D(0,1)$.

For all $\tau>0$ small enough, we set

$$
B^{\lceil}:=X^{\uparrow}\left(D\left(0, \tau^{3 / 4}\right)\right),
$$

and, for all $\rho>0$ satisfying

$$
\frac{1}{C} \tau \leq \rho^{2} \leq C \tau,
$$

for some fixed constant $C>1$, we define

$$
B_{j}^{\downarrow}:=X^{\downarrow}\left(D\left(\rho z_{j}, \tau^{3 / 4}\right)\right)
$$

for $j=0, \ldots, m$. We also define

$$
p^{\uparrow}:=X^{\uparrow}(0)
$$

to be the north pole of $S^{2}$, and, for $j=0, \ldots, m$, we define the points

$$
p_{j}^{\llcorner}:=X^{\downarrow}\left(\rho z_{j}\right),
$$

which are $m+1$ points arranged symmetrically near the south pole of $S^{2}$. By construction, $p_{j+1}^{\downarrow}$ is the image of $p_{j}^{\downarrow}$ by $\mathscr{R}_{m+1}$.

Definition 6.1. We define $\mathfrak{S}_{\tau, \rho}$ to be the surface obtained by excising from $S^{2}$ the sets $B^{\lceil}$and $B_{j}^{\downarrow}$ for $j=0, \ldots, m$.

Observe that, provided $\tau$ is chosen small enough, the surface $\mathfrak{S}_{\tau, \rho}$ has $m+2$ boundaries. Moreover, this surface has been constructed in such a way that it is invariant under the action of the dihedral group $\operatorname{Dih}_{m+1}^{(2)}$.

The mean curvature of vertical graphs. We recall some well-known facts about the mean curvature of vertical graphs in $\mathbb{R}^{3}$. The mean curvature of the graph of the function $u \in \mathscr{C}_{\text {loc }}^{2}\left(\mathbb{R}^{2}\right)$, namely, the surface parametrized by

$$
X(x):=(x, u(x)) \in \mathbb{R}^{3},
$$

where $x$ belongs to some open domain in $\mathbb{R}^{2}$, is given by

$$
M(u):=\frac{1}{2} \operatorname{div}\left(\frac{\nabla u}{\sqrt{1+|\nabla u|^{2}}}\right) .
$$

Recall that the mean curvature is defined to be the average of the principal curvatures; this explains the factor $\frac{1}{2}$. 
It follows from this formula that the linearized mean curvature operator about the graph of $u$ is given by

$$
\begin{aligned}
D M(u)(v)=\frac{\Delta v}{2 W}+ & \frac{3}{2 W^{5}}(\nabla u \cdot \nabla v) D^{2} u[\nabla u, \nabla u] \\
& \quad-\frac{1}{2 W^{3}}\left((\nabla u \cdot \nabla v) \Delta u+D^{2} v[\nabla u, \nabla u]+2 D^{2} u[\nabla u, \nabla v]\right),
\end{aligned}
$$

where

$$
W:=\sqrt{1+|\nabla u|^{2}}
$$

and where $D^{2} f[\cdot, \cdot]$ is the second order differential of the function $f$. One should be aware that $D M(u)$ is not the Jacobi operator $J_{u}$ about the graph of the function $u$, since nearby surfaces are not parametrized as normal graphs, but as vertical graphs over the horizontal plane. As explained in Section 2, this operator and the Jacobi operator are conjugate, and, in fact, assuming the vertical graph is oriented so that the unit normal vector field points upward, we have the relation

$$
X^{*}\left(J_{u} w\right)=D M(u)\left(W X^{*} w\right)
$$

for any function defined on the graph of $u$.

Of interest is the case where, for example,

$$
u(x)= \pm \sqrt{1-|x|^{2}}
$$

where $x=\left(x_{1}, x_{2}\right) \in \mathbb{R}^{2}$. According to the sign chosen, the graph of $u$ is the lower or the upper hemisphere of the sphere of radius 1 centered at the origin. In this case, we have

$$
\nabla u(x)=\mp \frac{x}{\sqrt{1-|x|^{2}}}, \quad \nabla^{2} u(x)=\mp \frac{\left(1-|x|^{2}\right) \mathrm{Id}+x \otimes x}{\left(1-|x|^{2}\right)^{3 / 2}}
$$

and

$$
\Delta u(x)=\mp \frac{2-|x|^{2}}{\left(1-|x|^{2}\right)^{3 / 2}} .
$$

Using these, we find that the explicit expression of $D H(u)$ in $D(0,1)$ is

$$
D M(u) w=\frac{1}{2}\left(1-|x|^{2}\right)^{1 / 2}\left(\Delta w-\nabla^{2} w(x, x)-4(x \cdot \nabla w)\right) .
$$

Green's function. Let $N_{0}$ denote the inward pointing unit normal vector field on $S^{2}$. We consider an inward pointing vector field $N_{0}^{\mathrm{b}}$, which is equal to $N_{0}$ close to the (horizontal) equator of $S^{2}$ and which is equal to a vertical unit vector field close to the north and south pole of $S^{2}$ (still pointing inward). 
We define $\mathbb{L}$ to be the linearized mean curvature operator using the vector field $N_{0}^{\mathrm{b}}$. According to the analysis in Section 2, we can write

$$
\llbracket w:=\frac{1}{2}\left(\Delta_{S^{2}}+2\right)\left(N_{0} \cdot N_{0}^{b} w\right) .
$$

We let $\Gamma_{\rho}$ be the unique solution of

$$
\mathbb{L} \Gamma_{\rho}=-\pi \delta_{p^{\downarrow}}-\frac{\pi}{\sqrt{1-\rho^{2}}} \frac{1}{m+1}\left(\delta_{p_{0}^{\downarrow}}+\cdots+\delta_{p_{m}}\right),
$$

which satisfies the orthogonality conditions

$$
\int_{S^{2}} x_{i} \Gamma_{\rho} d \mathrm{vol}_{S^{2}}=0
$$

for $i=1,2$ and 3. Here $\delta_{q}$ is the Dirac mass at the point $q$. The existence of $\Gamma_{\rho}$ is guaranteed by the fact that the distribution on the right side of (6-4) is orthogonal to the cokernel of $\mathbb{L}$. Indeed, the Jacobi operator is selfadjoint and its kernel and cokernel are equal and spanned by the restriction of the coordinate functions to the unit sphere. Thanks to (6-3), we conclude that the cokernel of $\mathbb{L}$ is also spanned by the restriction of the coordinate functions to the unit sphere, multiplied by the factor $N_{0} \cdot N_{0}^{b}$. For the sake of simplicity, we define the functions

$$
\tilde{x}_{i}:=x_{i} N_{0} \cdot N_{0}^{b}
$$

for $i=1,2,3$, which are obtained by multiplication of the coordinate functions by $N_{0} \cdot N_{0}^{b}$.

Now

$$
\left\langle x_{1}, \delta_{p^{\dagger}}\right\rangle_{\mathscr{D}, \mathscr{D}^{\prime}}=\left\langle x_{2}, \delta_{p^{\dagger}}\right\rangle_{\mathscr{D}, \mathscr{D}^{\prime}}=0,
$$

since both $x_{1}$ and $x_{2}$ vanish at the north pole of $S^{2}$ and

$$
\left\langle x_{1}, \delta_{\left.p_{j}\right\rangle_{\mathscr{D}, \mathscr{D}^{\prime}}}=\rho \cos \left(\frac{2 \pi}{m+1} j\right) \quad \text { and } \quad\left\langle x_{2}, \delta_{p_{j}^{\downarrow}}\right\rangle_{\mathscr{D}, \mathscr{D}^{\prime}}=\rho \sin \left(\frac{2 \pi}{m+1} j\right)\right. \text {. }
$$

Since

$$
\sum_{j=0}^{m} \cos \left(\frac{2 \pi}{m+1} j\right)=\sum_{j=0}^{m} \sin \left(\frac{2 \pi}{m+1} j\right)=0,
$$

we conclude that the distribution on the right side of (6-4) is orthogonal to the coordinate functions $x_{1}$ and $x_{2}$. Geometrically, this just follows from the fact that the points $p_{j}^{\llcorner}$are symmetrically arranged around the $x_{3}$-axis. Finally, we have

$$
\left\langle x_{3}, \delta_{p^{\uparrow}}\right\rangle_{\mathscr{D}, \mathscr{D}^{\prime}}=1 \quad \text { and } \quad\left\langle x_{3}, \delta_{p_{j}^{\downarrow}}\right\rangle_{\mathscr{D}, \mathscr{D}^{\prime}}=-\sqrt{1-\rho^{2}},
$$

and, again, we conclude that the distribution on the right side of (6-4) is orthogonal to the coordinate function $x_{3}$ thanks to the choice of the constant in front of the Dirac 
masses at the points $p_{j}^{L}$. Geometrically, this has some interesting consequences and can be interpreted as a conservation of the vertical flux of the surfaces we try to construct. We shall return to this point later on. Finally, observe that $\Gamma_{\rho}$ is invariant under the action of the elements of $\operatorname{Dih}_{m+1}^{(2)}$.

We now turn to the expansion of the function $\Gamma_{\rho}$ near the north pole of $S^{2}$.

Lemma 6.2. The expansion

$$
X^{\lceil *} \Gamma_{\rho}(x)=-\log |x|+a^{\uparrow}+\mathcal{O}_{\mathscr{C} \infty}\left(|x|^{2}\right)
$$

holds in a fixed neighborhood of 0 , where the constant $a^{\uparrow} \in \mathbb{R}$ depends smoothly on $\rho$ and is bounded as $\rho$ tends to 0 . Moreover, the estimate on $\mathcal{O}_{\dot{\varphi} \infty}\left(|x|^{2}\right)$ is uniform as $\rho$ tends to 0 .

Proof. We define the function $\Gamma_{0}$ on the upper hemisphere by

$$
X^{\vdash^{*}} \Gamma_{0}(x)=-\log |x|,
$$

and, using (6-2), we compute

$$
X^{\vdash^{*}}\left(\mathbb{L} \Gamma_{0}+\pi \delta_{p^{\dagger}}\right)=\frac{3}{2} \sqrt{1-|x|^{2}} .
$$

This immediately implies that, close to $p^{\uparrow}$, the function $\Gamma_{\rho}-\Gamma_{0}$ is smooth. In particular, this function can be expanded as

$$
X^{\vdash^{*}}\left(\Gamma_{\rho}-\Gamma_{0}\right)(x)=a^{\uparrow}+b^{\uparrow} \cdot x+\mathcal{O}_{\mathscr{e} \infty}\left(|x|^{2}\right),
$$

where $a^{\uparrow} \in \mathbb{R}$ and $b^{\lceil} \in \mathbb{R}^{2}$ depend smoothly on $\rho$ and remain bounded as $\rho$ tends to 0 . Since the function $\Gamma_{\rho}$ is also invariant under the action of the elements of $\operatorname{Dih}_{m+1}^{(2)}$, we conclude that necessarily $b^{\uparrow}=0$. This completes the proof.

Near the other poles, the function $\Gamma_{\rho}$ also has an expansion which we now describe. As can be suspected, this description relies on the expansion of the function

$$
G(x):=-\sum_{j=0}^{m} \log \left|x-\rho z_{j}\right|
$$

at any of its singularities. Since this is a key point in our construction, we spend some time deriving this expansion carefully. By symmetry, it is enough to expand this function at $\rho z_{0}$. We change variables and write $x=\rho z_{0}+y$. We then expand

$$
\log \left|y-\rho\left(z_{j}-z_{0}\right)\right|=\log \rho+\log \left|z_{j}-z_{0}\right|+\frac{1}{\rho} \frac{z_{0}-z_{j}}{\left|z_{0}-z_{j}\right|^{2}} \cdot y+\mathcal{O}\left(\frac{|y|^{2}}{\rho^{2}}\right) .
$$

Hence we find

$$
G\left(\rho z_{0}+y\right)=-\log |y|-m \log \rho-\sum_{j=1}^{m} \log \left|z_{j}-z_{0}\right|-\frac{1}{\rho} \sum_{j=1}^{m} \frac{z_{0}-z_{j}}{\left|z_{0}-z_{j}\right|^{2}} \cdot y+\mathcal{O}\left(\frac{|y|^{2}}{\rho^{2}}\right) .
$$


It is easy to check the identity

Setting

$$
\sum_{j=1}^{m} \frac{z_{0}-z_{j}}{\left|z_{0}-z_{j}\right|^{2}}=\frac{m}{2} z_{0} .
$$

$$
a_{0}^{\downarrow}:=\sum_{j=1}^{m} \log \left|z_{j}-z_{0}\right|,
$$

we can write

$$
G\left(\rho z_{0}+y\right)=-\log |y|-m \log \rho-a_{0}^{\llcorner}-\frac{m}{2 \rho} z_{0} \cdot y+\mathcal{O}\left(\frac{|y|^{2}}{\rho^{2}}\right) .
$$

Similar estimates can be obtained for the partial derivatives of $G$. Finally, we have

$$
\Delta G=-2 \pi\left(\delta_{\rho z_{0}}+\cdots+\delta_{\rho z_{m}}\right) .
$$

We now prove that, at $p_{j}^{\downarrow}$, the expansion of the function $X^{L *} \Gamma_{\rho}$ is (in some sense to be made precise) close to the expansion of $G$ near $\rho z_{j}$.

Lemma 6.3. The expansion

$$
X^{\mid *} \Gamma_{\rho}\left(\rho z_{j}+y\right)=-\frac{1}{m+1}\left(\log |y|+m \log \rho+a_{0, \rho}^{\downarrow}+\frac{m}{2 \rho} z_{j} \cdot y\right)+\mathscr{O}_{\mathscr{C} \infty}\left(\tau^{1 / 2}\right)
$$

holds for $|y| \in\left[\frac{1}{2} \tau^{3 / 4}, 2 \tau^{3 / 4}\right]$. Here $a_{0, \rho}^{\downarrow} \in \mathbb{R}$ smoothly depends on $\rho>0$ and is uniformly bounded as $\rho$ tends to 0 .

Proof. Thanks to the invariance with respect to the action of $\operatorname{Dih}_{m+1}^{(2)}$, it is enough to describe this expansion near the point $p_{0}^{\downarrow}$. As in the proof of Lemma 6.2, we show that, near the south pole of $S^{2}$, the function $X^{{ }^{*}} \Gamma_{\rho}$ is not too far from $G$. To this end, we define $\tilde{\Gamma}_{\rho}$ on the lower hemisphere of $S^{2}$ by

$$
X^{\llcorner *} \tilde{\Gamma}_{\rho}=G,
$$

and, thanks to (6-2), we can compute

$$
\begin{aligned}
& X^{L *}\left(\mathbb{L} \tilde{\Gamma}_{\rho}+\pi \sqrt{1-\rho^{2}}\left(\delta_{p_{0}^{\downarrow}}+\cdots+\delta_{p_{m}}\right)\right) \\
& =\frac{1}{2} \sqrt{1-|x|^{2}} \sum_{j=0}^{m}\left(3-2 \rho \frac{z_{j} \cdot\left(x-\rho z_{j}\right)}{\left|x-\rho z_{j}\right|^{2}}+\frac{\rho^{2}}{\left|x-\rho z_{j}\right|^{2}}\left(1-2 \frac{\left(z_{j} \cdot\left(x-\rho z_{j}\right)\right)^{2}}{\left|x-\rho z_{j}\right|^{2}}\right)\right) .
\end{aligned}
$$

Observe that the right side contains three terms which have different regularity properties. The first one is a smooth function which depends smoothly on $\rho$ and which is invariant by rotation. The second function has a singularity of order 1 at each $\rho z_{j}$ and is bounded by a constant times $\rho\left|x-\rho z_{j}\right|^{-1}$. The third function has a singularity of order 2 at each $\rho z_{j}$ and is bounded by a constant times $\rho^{2}\left|x-\rho z_{j}\right|^{-2}$. 
As a consequence, $X^{l *}\left(\tilde{\Gamma}_{\rho}-\Gamma_{\rho}\right)$ can be decomposed into the sum of three functions, which can be analyzed independently. The first one, $f_{\rho}^{(1)}$, is smooth in a fixed neighborhood of 0 and depends smoothly on the parameter $\rho$. This implies that, near each $\rho z_{j}$, this function has a Taylor expansion with coefficients smoothly depending on $\rho$. Hence

$$
f_{\rho}^{(1)}(x)=f_{\rho}^{(1)}\left(\rho z_{0}\right)+\nabla f_{\rho}^{(1)}\left(\rho z_{0}\right) \cdot\left(x-\rho z_{0}\right)+\mathcal{O}\left(\left|x-\rho z_{0}\right|^{2}\right) .
$$

Observe that $\nabla f_{\rho}^{(1)}(0)=0$, and hence $\left|\nabla f_{\rho}^{(1)}\left(\rho z_{0}\right)\right| \leq C \rho$. We conclude that $f_{\rho}^{(1)}(x)=f_{\rho}^{(1)}\left(\rho z_{0}\right)+\mathcal{O}\left(\tau^{5 / 4}\right)$ when $\left|x-\rho z_{0}\right| \in\left[\frac{1}{2} \tau^{3 / 4}, 2 \tau^{3 / 4}\right]$.

Since $\sum_{j}\left|z-z_{j}\right|^{-1} \in L^{p}\left(D\left(0, \frac{1}{2}\right)\right)$ for all $p \in(1,2)$, we find that the second function

$$
f_{\rho}^{(2)} \in W^{2, p}(D(0,1 / 3)),
$$

and hence it is continuous near $\rho z_{0}$ and $f_{\rho}^{(2)}(x)-f_{\rho}^{(2)}\left(\rho z_{0}\right)$ is bounded by a constant times $\rho \sum_{j=0}^{m}\left|x-\rho z_{j}\right|^{\nu}$ for any given $v<1$. In particular, $f_{\rho}^{(2)}(x)=$ $f_{\rho}^{(2)}\left(\rho z_{0}\right)+\mathcal{O}\left(\tau^{(2+3 v) / 4}\right)$ when $\left|x-\rho z_{0}\right| \in\left[\frac{1}{2} \tau^{3 / 4}, 2 \tau^{3 / 4}\right]$.

Finally, using Proposition 6.6, the third function $f_{\rho}^{(3)}$ is bounded by a constant times $\rho^{2} \sum_{j=0}^{m}\left|x-\rho z_{j}\right|^{\mu}$ for any $\mu \in(-1,0)$.

In particular, when $\left|x-\rho z_{0}\right| \in\left[\frac{1}{2} \tau^{3 / 4}, 2 \tau^{3 / 4}\right]$, we find that the sum of these functions can be decomposed as the sum of a constant function (smoothly depending on $\rho$ ) and a function which is bounded by a constant times $\tau^{1 / 2}$ (choose $v=\frac{1}{2}$ and $\mu=-\frac{1}{2}$ ). The statement then follows at once.

It is interesting to observe that $\Gamma_{\rho}$ depends on $\rho>0$ since the points $p_{j}^{\downarrow}$ do, and, as $\rho$ tends to 0 , the sequence $\Gamma_{\rho}$ converges on compact sets to the unique solution of

$$
\llbracket \Gamma_{0}=-\pi\left(\delta_{p^{\uparrow}}+\delta_{p} \downarrow\right),
$$

which is $L^{2}$-orthogonal to the smooth kernel of $\Delta_{S^{2}}+2$. Recall that $p^{\lceil}$denotes the north pole of $S^{2}$, and we now agree that $p^{\downarrow}$ denotes the south pole of $S^{2}$.

Remark 6.4. If a solution to $\mathbb{L} w=0$ is defined in $S^{2}-\left\{p^{\uparrow}, p^{\dagger}\right\}$, is invariant under the action of $\operatorname{Dih}_{m+1}^{(2)}$, and is bounded by a constant times $\operatorname{dist}\left(\cdot,\left\{p^{\uparrow}, p^{\dagger}\right\}\right)^{v}$ for some $v \in(-1,0)$, then it is a linear combination of $\tilde{x}_{3}$ and $\Gamma_{0}$. This will be useful later.

We now summarize the above analysis. We set

$$
u^{\uparrow}(x):=\sqrt{1-|x|^{2}} \text { and } u^{\downarrow}(x):=-\sqrt{1-|x|^{2}} .
$$

Observe that, thanks to the previous results, near 0 , the graph of the function

$$
v^{\urcorner}:=u^{\uparrow}+\tau X^{\lceil *} \Gamma_{\rho}
$$


can be expanded as

$$
v^{\uparrow}(x)=1+\tau\left(m \log \rho+a^{\lceil}\right)+\tau \log |x|+\mathcal{O}_{\dot{\wp} \infty}\left(\tau^{3 / 2}\right)
$$

for $|x| \in\left[(1 / 2) \tau^{3 / 4}, 2 \tau^{3 / 4}\right]$, where $a^{\uparrow} \in \mathbb{R}$ smoothly depends on $\rho$. Moreover, we see that, near $\rho z_{j}$, the graph of the function

$$
v^{\downarrow}:=u^{\downarrow}+\tau X^{L^{*}} \Gamma_{\rho}
$$

can be expanded as

$v^{L}\left(\rho z_{j}+y\right)$

$=-\sqrt{1-\rho^{2}}-\frac{\tau}{m+1}\left(m \log \rho+a^{\downarrow}\right)-\frac{\tau}{m+1} \log |y|-\left(\rho-\frac{m}{m+1} \frac{\tau}{2 \rho}\right) z_{j} \cdot y+\mathscr{O}_{\mathfrak{C} \infty}\left(\tau^{3 / 2}\right)$

for $|y| \in\left[(1 / 2) \tau^{3 / 4}, 2 \tau^{3 / 4}\right]$, where $a^{\downarrow} \in \mathbb{R}$ depends smoothly on $\rho$. The key point in our construction is that the constant in front of $z_{j} \cdot y$ can be adjusted by choosing $\rho$ appropriately. Indeed, if we define $\rho_{0}>0$ by the identity

$$
2(m+1) \rho_{0}^{2}=m \tau,
$$

then, when $\rho=\rho_{0}$, the constant in front of $z_{j} \cdot y$ in the last expansion is exactly 0 , while choosing $\rho \neq \rho_{0}$ slightly larger or smaller allows one to prescribe any value of this constant, close enough to 0 .

Mapping properties of the Jacobi operator about a punctured sphere. We first define on $S^{2}$ the distance function to the punctures $p^{\uparrow}, p_{0}^{\downarrow}, \ldots, p_{m}^{L}$ by

$$
d:=\operatorname{dist}_{S^{2}}\left(\cdot,\left\{p^{\uparrow}, p_{0}^{\downarrow}, \ldots, p_{m}^{\downarrow}\right\}\right) .
$$

Even though this is not apparent in the notation, the function $d$ depends implicitly on $\rho$, since it depends on the location of the points $p_{j}^{\downarrow}$, which themselves depend on $\rho$. We can define some weighted spaces on

$$
S^{*}:=S^{2}-\left\{p^{\uparrow}, p_{0}^{\downarrow}, \ldots, p_{m}^{\downarrow}\right\} .
$$

For $v \in \mathbb{R}$ and $k \in \mathbb{N}$, we define $\mathscr{C}_{v}^{k, \alpha}\left(S^{*}\right)$ to be the space of functions $w \in \mathscr{C}_{\text {loc }}^{k, \alpha}\left(S^{*}\right)$ for which the following norm is finite:

$$
\begin{aligned}
\|w\|_{\mathscr{C}_{v}^{k, \alpha}\left(S^{*}\right)}:=\sum_{j=0}^{k} \sup _{p \in S^{*}} d^{-v+j}(p)\left\|\nabla^{j} w(p)\right\|_{g_{S^{2}}} & \\
& \quad+\sup _{\zeta \in(0, \pi / 2)} \sup _{d(p), d(q) \in[\zeta, 2 \zeta]} \zeta^{-v+k+\alpha} \frac{\left\|\nabla^{k} w(p)-\nabla^{k} w(q)\right\|_{g_{S^{2}}}}{\operatorname{dist}_{S^{2}}(p, q)^{\alpha}} .
\end{aligned}
$$

We further assume that the functions in $\mathscr{C}_{v}^{k, \alpha}\left(S^{*}\right)$ are invariant under the action of $\operatorname{Dih}_{m+1}^{(2)}$. Again, the weighted spaces $\mathscr{C}_{v}^{k, \alpha}\left(S^{*}\right)$ do implicitly depend on $\rho$. 
We consider the operator

$$
\begin{aligned}
\mathbb{L}_{v}: \mathscr{C}_{v}^{2, \alpha}\left(S^{*}\right) & \rightarrow \mathscr{C}_{v-2}^{0, \alpha}\left(S^{*}\right), \\
w & \mapsto \mathbb{L} w .
\end{aligned}
$$

It is easy to check that $\mathbb{L}_{v}$ is well defined.

Recall that $\mathbb{L}$ is conjugate to $\Delta_{S^{2}}+2$. When acting on a smooth function defined on $S^{2}$, the mapping properties of $\Delta_{S^{2}}+2$ are well understood, and we recall that the kernel of this operator is spanned by the restriction to $S^{2}$ of the linear forms on $\mathbb{R}^{3}$. Since we are assuming that the functions we consider are invariant under the action of the dihedral group $\operatorname{Dih}_{m+1}^{(2)}$, this implies that the bounded kernel of $\mathbb{E}$ has dimension 1 . We now investigate the mapping properties of $\mathbb{L}$ (or, alternatively, $\Delta_{S^{2}}+2$ ) when acting on functions belonging to the weighted spaces we have just defined.

Proposition 6.5. Assume that $v \in(-1,0)$. Then there exist constants $C, \rho_{0}>0$, only depending on $v$, such that, for all $\rho \in\left(0, \rho_{0}\right)$, we have

$$
\|w\|_{\mathscr{C}_{v}^{2, \alpha}\left(S^{*}\right)} \leq C\|\mathbb{L} w\|_{\mathscr{C}_{v}^{0, \alpha}\left(S^{*}\right)},
$$

for all functions $w$ in the $L^{2}\left(S^{2}\right)$-orthogonal complement of the functions $\tilde{x}_{3}$ and $\Gamma_{\rho}$. Proof. As usual, thanks to Schauder's estimates, it is enough to prove that

$$
\left\|d^{-v} w\right\|_{L^{\infty}\left(S^{*}\right)} \leq C\left\|d^{2-v} \mathbb{L} w\right\|_{L^{\infty}\left(S^{*}\right)}
$$

for all $\rho$ small enough.

As usual, the proof of this estimate is by contradiction. Assume that the estimate is not true. Then there exists a sequence $\rho_{n}$ tending to 0 and a sequence of functions $w_{n}$ such that

$$
\left\|d^{-v} w_{n}\right\|_{L^{\infty}\left(S^{*}\right)}=1 \quad \text { and } \quad \lim _{n \rightarrow \infty}\left\|d^{2-v} \llbracket w_{n}\right\|_{L^{\infty}\left(S^{*}\right)}=0 .
$$

Moreover $w_{n}$ is invariant under the action of $\operatorname{Dih}_{m+1}^{(2)}$ and is $L^{2}$-orthogonal to $\tilde{x}_{3}$ and $\Gamma_{\rho_{n}}$ (recall that $\Gamma_{\rho}=\Gamma_{\rho_{n}}$ depends on $\rho_{n}$ ). Hence

$$
\int_{S^{2}} \tilde{x}_{3} w_{n} d \mathrm{vol}_{S^{2}}=0
$$

and

$$
\int_{S^{2}} \Gamma_{\rho_{n}} w_{n} d \mathrm{vol}_{S^{2}}=0
$$

We choose a point $q_{n} \in S^{*}$ such that

$$
\left|w_{n}\left(q_{n}\right)\right| \geq \frac{1}{2} d^{v}\left(q_{n}\right)
$$


and we distinguish various cases according to the behavior of the sequence $d\left(q_{n}\right)$. In each case, we rescale coordinates (using the exponential map) by $1 / d\left(q_{n}\right)$, and we use elliptic estimates together with the Ascoli-Arzelà theorem to extract from the sequence $\tilde{w}_{n}:=d^{-v}\left(q_{n}\right) w_{n}$ convergent subsequences. Finally, we pass to the limit in the equation satisfied by $\tilde{w}_{n}$. If, for some subsequence, $d\left(q_{n}\right)$ remains bounded away from 0 , we get in the limit a nontrivial solution of

$$
\llbracket w=0,
$$

which is defined in $S^{2}-\left\{p^{\uparrow}, p^{\downarrow}\right\}$, where we recall that $p^{\uparrow}$ and $p^{\downarrow}$ denote the north and south poles of $S^{2}$. Moreover, $w$ is bounded by a constant times $\left(\operatorname{dist}\left(p,\left\{p^{\uparrow}, p^{\dagger}\right\}\right)\right)^{v}$ and $w$ is invariant under the action of $\operatorname{Dih}_{m+1}^{(2)}$. Finally, we can pass to the limit in (6-5) and (6-6) and check that $w$ is $L^{2}$-orthogonal to $\tilde{x}_{3}$ and $\Gamma_{0}:=\lim _{n \rightarrow \infty} \Gamma_{\rho_{n}}$. It is easy to check (see Remark 6.4) that this implies that $w \equiv 0$, which is a contradiction.

The second case we have to consider is the case where $\lim _{n \rightarrow \infty} d\left(q_{n}\right)=0$ and $\lim _{n \rightarrow \infty} d\left(q_{n}\right) / \rho_{n}=+\infty$ or the case where $\lim _{n \rightarrow \infty} d\left(q_{n}\right) / \rho_{n}=0$. In either case, we obtain a nontrivial solution of

$$
\Delta w=0
$$

in $\mathbb{R}^{2}-\{0\}$, which is bounded by a constant times $\operatorname{dist}(\cdot,\{0\})^{\nu}$. It is easy to check that $w \equiv 0$ since $\delta \notin \mathbb{Z}$, which is again a contradiction.

Finally, we consider the case where $\lim _{n \rightarrow \infty} d\left(q_{n}\right) / \rho_{n}$ exists. In this case, we obtain a nontrivial solution of $\Delta w=0$ in $\mathbb{R}^{2}-\left\{r_{0} z_{0}, \ldots, r_{0} z_{m}\right\}$ for some $r_{0}>0$. Moreover, we know that this solution is bounded by a constant times $\left(\operatorname{dist}\left(\cdot,\left\{r_{0} z_{0}, \ldots, r_{0} z_{m}\right\}\right)\right)^{v}$ and $w$ is also invariant under the action of $\operatorname{Dih}_{m+1}^{(2)}$. Inspection of the behavior of $w$ at the points $r_{0} z_{j}$ together with the fact that $v>-1$ and $w$ is invariant with respect to the action of $\operatorname{Dih}_{m+1}^{(2)}$ implies that $w$ is a solution in the sense of distributions of

$$
\Delta w=a \sum_{j=0}^{m} \delta_{r_{0} z_{j}}
$$

for some $a \in \mathbb{R}$. Then inspection of $w$ at infinity together with the fact that $v<0$ implies that $a=0$, and hence $w \equiv 0$. This is again a contradiction.

Thanks to the previous result, we can prove:

Proposition 6.6. Assume that $v \in(-1,0)$ is fixed. Then the operator $\mathbb{L}_{v}$ is surjective and has a 2-dimensional kernel spanned by the functions $\tilde{x}_{3}$ and $\Gamma_{\rho}$. Moreover, the right inverse of $\mathbb{L}_{v}$, which is chosen so that its image is in the $L^{2}$-orthogonal complement of the kernel of $\mathbb{L}_{v}$, has a norm which is bounded independently of $\rho$ small enough. 
Proof. The existence of a right inverse follows from the general theory developed, for example, in [Pacard 2008]. Nevertheless, we give a self-contained proof.

Let us assume that we are given a function $f \in \mathscr{C}^{0, \alpha}\left(S^{*}\right)$ which has compact support in $S^{*}$. Recall that the functions we are interested in are invariant under the action of $\operatorname{Dih}_{m+1}^{(2)}$. We choose $a \in \mathbb{R}$ so that $f-a \delta_{p}$ is orthogonal to the function $\tilde{x}_{3}$. In particular, this implies that we can solve

$$
\llbracket \tilde{w}=f-a \delta_{p^{\dagger}},
$$

and, choosing the constant $b \in \mathbb{R}$ appropriately, we can assume that $w:=\tilde{w}-b \Gamma_{\rho}$ is $L^{2}$-orthogonal to the function $\tilde{x}_{3}$ and $\Gamma_{\rho}$. Observe that

$$
\llbracket w=f
$$

in $S^{*}$, and also that $w \in \mathscr{C}_{v}^{2, \alpha}\left(S^{*}\right)$. In particular Proposition 6.5 applies and we have

$$
\|w\|_{\mathscr{C}_{v}^{2, \alpha}\left(S^{*}\right)} \leq C\|\mathbb{L} w\|_{\mathscr{C}_{v}^{0, \alpha}\left(S^{*}\right)} .
$$

The general result, when $f$ is not assumed to have compact support in $S^{*}$, can be handled as usual, using a sequence of functions having compact support and converging on compact sets to a given function in $\mathscr{C}_{v}^{0, \alpha}\left(S^{*}\right)$.

A third fixed-point argument. Assume that we are given $\tau, \tilde{\tau}>0$ small enough and satisfying

$$
|\tilde{\tau}-\tau| \leq \kappa \tau^{3 / 2},
$$

where the constant $\kappa>0$ is large enough (it will be fixed in Section 7). We also assume that $\rho>0$ satisfies

$$
\left|\rho-\frac{m}{m+1} \frac{\tau}{2 \rho}\right| \leq \kappa \tau^{3 / 4}
$$

We prove the existence of an infinite-dimensional family of constant mean curvature surfaces which are close to $\mathfrak{S}_{\tau, \rho}$ and are parametrized by their boundary values described by two functions $f^{\dagger}: S^{1} \rightarrow \mathbb{R}$ and $f^{\downarrow}: S^{1} \rightarrow \mathbb{R}$. The surfaces also depend on $\tilde{\tau}$ and $\rho$ satisfying the above estimates.

Proposition 6.7. Assume we are given $\kappa>0$ large enough (to be fixed later). For all $\tau, \tilde{\tau}>0$ small enough satisfying (6-7) and for all functions $f^{\lceil}$which are invariant under the action of the dihedral group $\operatorname{Dih}_{m+1}^{(2)}$ and $f^{\downarrow}$, which are invariant under the action of $\Psi_{2}$, satisfying both (H1) and

$$
\|f\|_{\mathscr{C}^{2, \alpha}\left(S^{1}\right)} \leq \kappa \tau^{3 / 2},
$$

there exists a constant mean curvature surface $\mathfrak{S}_{\tilde{\tau}, \rho, f} f^{\downarrow} \downarrow$ which is a graph over $\mathfrak{S}_{\tau, \rho}$, has $m+2$ boundaries (one boundary close to the north pole and $m+1$ 
boundaries close to the south pole), and is invariant under the action of the dihedral group $\operatorname{Dih}_{m+1}^{(2)}$. Close to the upper boundary, the surface $\mathfrak{S}_{\tilde{\tau}, \rho, f^{\vdash}, f \downarrow}$ is a vertical graph over the annulus

$$
\left\{x \in \mathbb{R}^{2}: \tau^{3 / 4} \leq|x| \leq 2 \tau^{3 / 4}\right\},
$$

for some function $x \mapsto V_{\tilde{\tau}, \rho, f^{\dagger}, f^{\downarrow}}^{\uparrow}\left(\tau^{-3 / 4} x\right)$ which can be expanded as

(6-9) $\quad V_{\tilde{\tau}, \rho, f^{\uparrow}, f^{\downarrow}}^{\uparrow}(x)$

$=1+\tilde{\tau}\left(m \log \rho+a_{\tilde{\tau}, \rho, f^{\dagger}, f^{\downarrow}}^{\dagger}\right)+\frac{3}{4} \tilde{\tau} \log \tau+\tilde{\tau} \log |x|-W_{f^{\dagger}}^{\text {out }}(x)+\bar{V}_{\tilde{\tau}, \rho, f^{\dagger}, f^{\downarrow}}^{\uparrow}(x)$,

where $a^{\uparrow} \in \mathbb{R}, W_{f}^{\text {out }}$ denotes the bounded harmonic extension of $f$ in $\mathbb{R}^{2}-\bar{D}(0,1)$, and

$$
\left\|\bar{V}_{\tilde{\tau}, \rho, 0,0}^{\uparrow}\right\|_{\mathscr{C}^{2, \alpha}(\bar{D}(0,2)-D(0,1))} \leq C \tau^{3 / 2},
$$

and, given $v \in(-1,0)$,

$$
\begin{aligned}
& \left\|\bar{V}_{\tilde{\tau}, \rho, f^{\dagger}, f \downarrow}^{\uparrow}-\hat{V}_{\tilde{\tau}, \rho, f^{\prime \prime}, ', f l,}^{\dagger}\right\|_{\mathscr{C}^{2, \alpha}\left(\bar{D}(0,1)-D\left(0, \frac{1}{2}\right)\right)} \\
& \quad \leq C \tau^{(1+v) / 4}\left(\left\|f^{\left.\right|^{\prime}}-f^{\dagger}\right\|_{\mathscr{C}^{2, \alpha}\left(S^{1}\right)}+\left\|f^{\nu}-f^{\downarrow}\right\|_{\mathscr{C}^{2, \alpha}\left(S^{1}\right)}\right)
\end{aligned}
$$

for some constant $C>0$ independent of $\kappa, \tilde{\tau}$, and $f^{\uparrow}, f^{\downarrow}, f^{\rangle^{\prime}}, f^{\downarrow^{\prime}}$.

Near one of the lower boundaries, the surface $\mathscr{S}_{\tilde{\tau}, \rho, f^{\vdash}, f \downarrow}$ is a vertical graph over the annulus

$$
\left\{x \in \mathbb{R}^{2}: \tau^{3 / 4} \leq\left|x-\rho z_{0}\right| \leq 2 \tau^{3 / 4}\right\}
$$

for some function $x \mapsto V_{\tilde{\tau}, \rho, f^{\dagger}, f^{\downarrow}}^{\downarrow}\left(\tau^{3 / 4}\left(x-\rho z_{0}\right)\right)$, which can be expanded as

$$
\begin{aligned}
& V_{\tilde{\tau}, \rho, f^{\dagger}, f^{\downarrow}}^{\downarrow}(x) \\
& =-\sqrt{1-\rho^{2}}-\frac{\tilde{\tau}}{m+1}\left(m \log \rho+a_{\tilde{\tau}, \rho, f^{\vdash}, f \downarrow}^{\downarrow}\right)-\frac{3 \tilde{\tau}}{4(m+1)} \log \tau-\frac{\tilde{\tau}}{m+1} \log |x| \\
& \quad-\tau^{3 / 4}\left(\rho-\frac{m}{m+1} \frac{\tilde{\tau}}{2 \rho}\right) z_{0} \cdot x+W_{f^{\downarrow}}^{\text {out }}(x)+\bar{V}_{\tilde{\tau}, f^{\dagger}, f^{\downarrow}}^{\downarrow}(x),
\end{aligned}
$$

where $\bar{V}_{\tilde{\tau}, \rho, f^{\dagger}, f \downarrow}^{L}$ enjoys properties similar to those described above for $\bar{V}_{\tilde{\tau}, \rho, f^{\dagger}, f \downarrow}^{\downarrow}$. Moreover, both depend continuously on $\tilde{\tau}$ and $\rho$.

Proof. Again the arguments of the proof are similar to those in the previous sections. The equation we must solve can be written formally as

$$
\mathbb{L}\left(\tilde{\tau} \Gamma_{\rho}+\hat{F}+w\right)=Q\left(\tilde{\tau} \Gamma_{\rho}+\hat{F}+w\right),
$$


where $Q$ collects all the nonlinear terms. Here $\hat{F}$ is a function which can be described as follows: near the north pole $p^{\dagger}$,

$$
X^{\uparrow *} \hat{F}(x)=\chi W_{f^{\uparrow}}^{\text {out }}\left(\tau^{-3 / 4} x\right),
$$

where $\chi$ is a cutoff function identically equal to 1 in $D(0,1 / 4)$ and identically equal to 1 outside $D\left(0, \frac{1}{2}\right)$. Near the south pole, $p^{\downarrow}$

$$
X^{L^{*}} \hat{F}(x)=\sum_{j=0}^{m} \bar{\chi}\left(\frac{x-\rho z_{j}}{\rho}\right) W_{f^{\uparrow}}^{\text {out }}\left(x-\rho z_{j}\right),
$$

where $\bar{\chi}$ is a cutoff function identically equal to 1 in $D(0, c)$ and identically equal to 1 outside $D(0, c / 2)$. Here $c=\sin (\pi /(m+1))$ so that the balls of radius $c$ centered at the points $z_{j}$ for $j=0, \ldots, m$ are disjoint.

We choose an extension operator

$$
\hat{\mathscr{E}}_{\tau}: \mathscr{C}^{0, \alpha}\left(\mathfrak{S}_{\tau, \rho}\right) \rightarrow \mathscr{C}^{0, \alpha}\left(S^{*}\right)
$$

such that

$$
\hat{\mathscr{E}}_{\tau}(\psi)= \begin{cases}\psi & \text { in } \mathfrak{S}_{\tau, \rho}, \\ 0 & \text { in } X^{\uparrow}\left(D\left(0, \tau^{3 / 4} / 2\right)\right) \cup \bigcup_{j=0}^{m} X^{\downarrow}\left(D\left(\rho z_{j}, \tau^{3 / 4} / 2\right)\right),\end{cases}
$$

and

$$
\left\|\hat{\mathscr{E}}_{\tau}(\psi)\right\|_{\mathscr{C}_{v}^{0, \alpha}\left(S^{*}\right)} \leq C\|\psi\|_{\mathscr{C}_{v}^{0, \alpha}\left(\mathfrak{S}_{\tau, \rho}\right)} .
$$

By definition, the norm in the space $\mathscr{C}_{v}^{0, \alpha}\left(\mathfrak{S}_{\tau, \rho}\right)$ is defined exactly as the norm in $\mathscr{C}_{v}^{0, \alpha}\left(S^{*}\right)$, but points are restricted to $\mathfrak{S}_{\tau, \rho}$ instead of $S^{*}$.

We rewrite (6-13) as

$$
\llbracket w=\hat{\mathscr{E}}_{\tau}\left(-\mathbb{L} \hat{F}+Q\left(\tilde{\tau} \Gamma_{\rho}+\hat{F}+w\right)\right) .
$$

Observe that, by construction, $\mathbb{\complement}\left(\tilde{\tau} \Gamma_{\rho}\right)=0$ away from the singular points.

Again, on the right side, it is understood that we consider the image by $\hat{\mathscr{E}}_{\tau}$ of the restriction of the functions to $\mathfrak{S}_{\tau, \rho}$.

We assume that $v \in(-1,0)$ is fixed. It is easy to check that there exists a constant $c>0$ (independent of $\kappa$ ) and a constant $c_{\kappa}>0$ (depending on $\kappa$ ) such that

$$
\begin{aligned}
\left\|\hat{\mathscr{E}}_{\tau}\left(Q\left(\tilde{\tau} \Gamma_{\rho}\right)\right)\right\|_{\mathscr{C}_{v-2}^{2, \alpha}\left(S^{*}\right)} & \leq c \tau^{(6-3 v) / 4}, \\
\left\|\hat{\mathscr{E}}_{\tau}(\mathbb{L} \hat{F})\right\|_{\mathscr{C}_{v-2}^{2, \alpha}\left(S^{*}\right)} & \leq c \tau^{(1-2 v) / 4}\left(\left\|f^{\dagger}\right\|_{\mathscr{C}^{2, \alpha}\left(S^{1}\right)}+\left\|f^{\downarrow}\right\|_{\mathscr{C}^{2, \alpha}\left(S^{1}\right)}\right),
\end{aligned}
$$

and

$$
\begin{aligned}
& \left\|\hat{\mathscr{E}}_{\tau}\left(Q\left(\tilde{\tau} \Gamma_{\rho}+\hat{F}^{\prime}+w^{\prime}\right)-Q\left(\tilde{\tau} \Gamma_{\rho}+\hat{F}+w\right)\right)\right\|_{\mathscr{C}_{v-2}^{2, \alpha}\left(S^{*}\right)} \\
& \quad \leq c_{\kappa}\left(\tau\left\|w^{\prime}-w\right\|_{\mathscr{C}_{v}^{2, \alpha}\left(S^{*}\right)}+\tau^{(4-3 v) / 4}\left(\left\|f^{\dagger \prime}-f^{\dagger}\right\|_{\mathscr{C}^{2, \alpha}\left(S^{1}\right)}+\left\|f^{\prime \prime}-f^{\downarrow}\right\|_{\mathscr{C}^{2, \alpha}\left(S^{1}\right)}\right)\right),
\end{aligned}
$$


provided $w$ and $w^{\prime}$ satisfy

$$
\|w\|_{\mathscr{C}_{v}^{2, \alpha}\left(S^{*}\right)}+\left\|w^{\prime}\right\|_{\mathscr{C}_{v}^{2, \alpha}\left(S^{*}\right)} \leq C \tau^{(6-3 v) / 4}
$$

for some fixed constant $C>0$ independent of $\kappa$. Here $\hat{F}$ and $\hat{F}^{\prime}$ are associated to the harmonic extensions of the boundary data $f^{\dagger}, f^{\downarrow}$ and $f^{l^{\prime}}, f^{l^{\prime}}$, respectively.

Now we make use of Proposition 6.6 to rephrase the problem as a fixed-point problem, and the previous estimates are precisely enough to solve this nonlinear problem using a fixed-point argument for contraction mappings in the ball of radius $C_{\kappa} \tau^{(6-3 v) / 4}$ in $\mathscr{C}_{v}^{2, \alpha}\left(S^{*}\right)$, where $C_{\kappa}$ is fixed large enough. Then, for all $\tau>0$ small enough, we find that there exists a constant $C_{\kappa}>0$ (depending on $\kappa$ ) such that, for all functions $f^{\downarrow}, f^{\downarrow}$ satisfying the above hypothesis, there exists a solution $w$ of (6-13) satisfying

$$
\|w\|_{\mathscr{C}_{v}^{2, \alpha}\left(S^{*}\right)} \leq C \tau^{(6-3 v) / 4} .
$$

In addition, we have the estimate

$$
\left\|w^{\prime}-w\right\|_{\mathscr{C}_{v}^{2, \alpha}\left(S^{*}\right)} \leq C_{\kappa} \tau^{(1-2 v) / 4}\left(\left\|f^{\downarrow^{\prime}}-f^{\lceil}\right\|_{\mathscr{C}^{2, \alpha}\left(S^{1}\right)}+\left\|f^{\downarrow^{\prime}}-f^{\downarrow}\right\|_{\mathscr{C}^{2, \alpha}\left(S^{1}\right)}\right)
$$

for some constant $C>0$, which does not depend on $\kappa$ or $\tau$, where $w$ (respectively $w^{\prime}$ ) is the solution associated to $f^{\dagger}, f^{\downarrow}$ (respectively $f^{\prime \prime}, f^{l^{\prime}}$ ).

The solution of (6-13) is obtained through a fixed-point theorem for contraction mappings, and it is classical to check that the solution we obtain depends continuously on the parameters of the construction. In particular, the constant mean curvature surface we obtain depends continuously on $\tilde{\tau}$ and $\rho$.

\section{Connecting the pieces together}

We keep the notation of the previous sections. We assume that $\kappa>0$ is large enough (to be chosen shortly) and assume that $\tau>0$ is chosen small enough so that all the results proven so far apply.

For all $\tilde{x} \in \mathbb{R}^{2}$, we define the annuli

$$
\begin{aligned}
& A_{\tau}^{\text {out }}(\tilde{x}):=\left\{x \in \mathbb{R}^{2}: \tau^{3 / 4} \leq|x-\tilde{x}| \leq 2 \tau^{3 / 4}\right\}, \\
& A_{\tau}^{\text {ins }}(\tilde{x}):=\left\{x \in \mathbb{R}^{2}: \frac{1}{2} \tau^{3 / 4} \leq|x-\tilde{x}| \leq \tau^{3 / 4}\right\} .
\end{aligned}
$$

Recall from page 137 the conditions (H1) and (H2). Also recall that a function $f$ defined on $S^{1}$ is invariant under the action of $\operatorname{Dih}_{m+1}^{(2)}$ if

$$
f\left(\theta+\frac{2 \pi}{m+1}\right)=f(\theta) \text { for all } \theta \in S^{1},
$$

and $f$ is invariant under the action of the symmetry $\mathscr{T}_{2}$ if

$$
f(-\theta)=f(\theta) \text { for all } \theta \in S^{1} .
$$


We now describe the different pieces of constant mean curvature surfaces we have at hand.

(i) Assume that we are given $f^{\uparrow} \in \mathscr{C}^{2, \alpha}\left(S^{1}\right)$, which is invariant under the action of $\operatorname{Dih}_{m+1}^{(2)}$ and satisfies (H1) and

$$
\left\|f^{\uparrow}\right\|_{\mathscr{C}^{2, \alpha}\left(S^{1}\right)} \leq \kappa \tau^{3 / 2} .
$$

Proposition 4.12 provides a constant mean curvature (equal to 1 ) surface $\mathfrak{D}_{\tau, f}^{+}$which is invariant under the action of the dihedral group $\operatorname{Dih}_{m+1}^{(2)}$, has one end asymptotic to the end of $\mathfrak{D}_{\tau}^{+}$, and which, close to its boundary, can be parametrized as the vertical graph of $x \mapsto U^{\uparrow}\left(\tau^{-3 / 4} x\right)$ over $A_{\tau}^{\text {ins }}(0)$, where

$$
U^{\uparrow}(x)=c^{\uparrow}+\tau \log |x|-W_{f}^{\text {ins }}(x)+\bar{U}^{\uparrow}(x),
$$

where

$$
c^{\uparrow}:=\tau \log \frac{2}{\tau^{1 / 4}} \in \mathbb{R},
$$

and where $\bar{U}^{\uparrow}$ satisfies (4-17) and (4-18). To simplify the notation we have not mentioned the data $\tau, f$ in the notation for $U^{\uparrow}$ and $\bar{U}^{\uparrow}$.

(ii) Next, we assume that we are given $\tau_{1}>0$ satisfying

$$
\left|\tau_{1}-\tau\right| \leq \kappa \tau^{3 / 2}
$$

and $\rho_{1}>0$ satisfying

$$
\left|\rho_{1}-\frac{m}{m+1} \frac{\tau}{2 \rho_{1}}\right| \leq \kappa \tau^{3 / 4}
$$

Further assume that we are given a function $f_{1}^{\uparrow} \in \mathscr{C}^{2, \alpha}\left(S^{1}\right)$ invariant under the action of the dihedral group $\operatorname{Dih}_{m+1}^{(2)}$ and a function $f_{1}^{\downarrow} \in \mathscr{C}^{2, \alpha}\left(S^{1}\right)$ invariant under the action of the symmetry $\mathscr{S}_{2}$, both satisfying (H1) and

$$
\left\|f_{1}^{\uparrow}\right\|_{\mathscr{C}^{2, \alpha}\left(S^{1}\right)} \leq \kappa \tau^{3 / 2} \text { and }\left\|f_{1}^{\downarrow}\right\|_{\mathscr{C}^{2, \alpha}\left(S^{1}\right)} \leq \kappa \tau^{3 / 2} .
$$

Proposition 6.7 provides a constant mean curvature (equal to 1) surface $\mathfrak{S}_{\tau_{1}, \rho_{1}, f_{1}^{\dagger}, f_{1}^{\downarrow}}$ which is invariant under the action of the dihedral group $\operatorname{Dih}_{m+1}^{(2)}$ and which, close to its upper boundary, can be parametrized as the vertical graph of $x \mapsto V^{\uparrow}\left(\tau^{-3 / 4} x\right)$ over $A_{\tau}^{\text {out }}(0)$, where

$$
\begin{aligned}
& V^{\uparrow}(x)=1+d^{\uparrow}+\tau_{1} \log |x|-W_{f_{1}^{\dagger}}^{\text {out }}(x)+\bar{V}^{\uparrow}(x), \\
& d^{\lceil}:=\tau_{1}\left(m \log \rho_{1}+a_{\tau_{1}, \rho, f_{1}^{\uparrow}, f_{1}^{\downarrow}}^{\uparrow}+\frac{3}{4} \log \tau\right) \in \mathbb{R},
\end{aligned}
$$

and where $\bar{V}^{\uparrow}$ satisfies (6-10) and (6-11). Close to one of its lower boundaries, this surface can be parametrized as a vertical graph for some function

$$
x \mapsto V^{\downarrow}\left(\tau^{-3 / 4}\left(x-\rho_{1} z_{0}\right)\right)
$$


over $A_{\tau}^{\text {out }}\left(\rho_{1} z_{0}\right)$ which can be expanded as

$$
\begin{aligned}
V^{\downarrow}(x) & =-1+c^{\downarrow}-\frac{\tau_{1}}{m+1} \log |x|-\tau^{3 / 4}\left(\rho_{1}-\frac{m}{m+1} \frac{\tau_{1}}{2 \rho}\right) z_{0} \cdot x+W_{f_{1}^{\prime}}^{\text {out }}(x)+\bar{V}^{\downarrow}(x), \\
c^{\downarrow} & :=1-\sqrt{1-\rho_{1}^{2}}-\frac{\tau_{1}}{m+1}\left(m \log \rho_{1}+a_{\left.\tau_{1}, \rho_{1}, f_{1}^{\downarrow}, f_{1}^{\downarrow}-\frac{3}{4} \log \tau\right) \in \mathbb{R},}\right.
\end{aligned}
$$

and where $\bar{V}^{l}$ satisfies estimates of the form (6-10) and (6-11). Again, to simplify the notation we have not mentioned the parameters $\tau_{1}, \rho_{1}, f_{1}^{\uparrow}, f_{1}^{\downarrow}$ in the notation for $V^{\uparrow}, \bar{V}^{\uparrow}, V^{l}$, and $\bar{V}^{l}$.

(iii) Assume that we are given $\tau_{2}>0$ satisfying

$$
\left|\tau_{2}-\frac{\tau}{m+1}\right| \leq \kappa \tau^{3 / 2},
$$

and a function $f_{2}^{\downarrow} \in \mathscr{C}^{2, \alpha}\left(S^{1}\right)$ which satisfies (H1), (H2), and

$$
\left\|f_{2}^{\downarrow}\right\|_{\mathscr{C}^{2, \alpha}\left(S^{1}\right)} \leq \kappa \tau^{3 / 2} .
$$

Proposition 5.5 provides a constant mean curvature (equal to 1 ) surface $\mathfrak{C}_{\tau_{2}, f_{2}}$ which is invariant under the action of $\mathscr{S}_{3}$, the symmetry with respect to the horizontal plane $x_{3}=0$, and is also invariant under the action of $\mathscr{Y}_{2}$, the symmetry with respect to the plane $x_{2}=0$. Moreover, close to its lower boundary, this surface can be parametrized as the vertical graph of $x \mapsto U^{L}\left(\tau^{-3 / 4} x\right)$ over $A_{\tau}^{\text {ins }}(0)$, where

$$
U^{\downarrow}(x)=d^{\downarrow}-\tau_{2} \log |x|+W_{f_{2}^{\perp}}^{\text {ins }}(x)+\bar{U}^{\downarrow}(x),
$$

where

$$
d^{l}:=-\tau_{2} \log \frac{2 \tau^{3 / 4}}{\tau_{2}} \in \mathbb{R}
$$

and where $\bar{U}^{l}$ satisfies (5-8) and (5-9). To simplify the notation we have not mentioned the data $\tau_{2}, f_{2}^{\downarrow}$ in the notation for $\bar{U}^{\downarrow}$ and $\bar{U}^{\downarrow}$.

Let us emphasize that the functions $f_{1}^{\downarrow}, f_{2}^{\downarrow}$ and $f^{\uparrow}, f_{1}^{\uparrow}$ are all assumed to satisfy (H1). Hence they have no constant term in their Fourier series. The function $f_{2}$ is also assumed to satisfy (H2). Now, the functions $f^{\uparrow}$ and $f_{1}^{\uparrow}$ are assumed to be invariant under the action of the dihedral group $\operatorname{Dih}_{m+1}^{(2)}$, and, as was already mentioned, this implies that both functions also satisfies $(\mathrm{H} 2)$ since its Fourier series does not contain any term of the form $z \cdot x$. Therefore, $f_{1}^{l}$ is the only function which does not satisfy (H2). Since $f_{1}^{\downarrow}$ is assumed to be invariant under the action of $\mathscr{Y}_{2}$, we can decompose it as

$$
f_{1}^{\downarrow}=\lambda_{1} z_{0} \cdot x+f_{1}^{\lfloor, \perp},
$$

where $\lambda_{1} \in \mathbb{R}$ and where $f_{1}^{\ell, \perp}$ satisfies both (H1) and (H2).

We denote by $\mathfrak{C}_{\tau_{2}, f_{2}, \rho_{1}}^{(0)}$ the surface $\mathfrak{C}_{\tau_{2}, f_{2}^{\downarrow}}$ translated by $\rho_{1} z_{0}$. For $j=1, \ldots, m$, 


$$
\mathfrak{C}_{\tau_{2}, f_{2}, \rho_{1}}^{(0)}:=\mathfrak{C}_{\tau_{2}, f_{2}^{\downarrow}}+\rho_{1} z_{0} .
$$

We denote the image of $\mathfrak{C}_{\tau_{2}, f_{2}, \rho_{1}}^{(0)}$ under the rotation $\left(\mathscr{R}_{m+1}\right)^{j}$ by

$$
\mathfrak{C}_{\tau_{2}, f_{2}^{\downarrow}, \rho_{1}}^{(j)}:=\left(\Re_{m+1}\right)^{j}\left(\mathfrak{C}_{\tau_{2}, f_{2}, \rho_{1}}^{(0)}\right) .
$$

In particular, the collection of surfaces $\mathfrak{C}_{\tau_{2}, f_{2}, \rho_{1}}^{(0)}, \ldots, \mathfrak{C}_{\tau_{2}, f_{2}^{\downarrow}, \rho_{1}}^{(m)}$ constitute $m+1$ constant mean curvature surfaces which are symmetric with respect to the dihedral group $\operatorname{Dih}_{m+1}^{(3)}$.

Given $t_{1} \in \mathbb{R}$ small enough, we denote by $\mathfrak{S}_{\tau_{1}, \rho_{1}, f_{1}, f_{1}^{\downarrow}, t_{1}}$ the surface $\mathfrak{S}_{\tau_{1}, \rho_{1}, f_{1}^{\dagger}, f_{1}^{\downarrow}}$ translated in the vertical direction by $\left(1-c^{\downarrow}+d^{\downarrow}+t_{1}\right) e_{3}$ :

$$
\mathfrak{S}_{\tau_{1}, \rho_{1}, f_{1}^{\downarrow}, f_{1}^{\downarrow}, t_{1}}:=\mathfrak{S}_{\tau_{1}, \rho_{1}, f_{1}^{\uparrow}, f_{1}^{\downarrow}}+\left(1-c^{\downarrow}+d^{\downarrow}+t_{1}\right) e_{3} .
$$

This is a constant mean curvature surface which is symmetric with respect to the dihedral group $\operatorname{Dih}_{m+1}^{(2)}$. Observe that the lower boundaries of $\mathfrak{C}_{\tau_{2}, f_{2}, \rho_{1}}^{(0)}, \ldots, \mathfrak{C}_{\tau_{2}, f_{2}, \rho_{1}}^{(m)}$ are close to the lower boundaries of $\mathfrak{S}_{\tau_{1}, \rho_{1}, f_{1}, f_{1}^{\downarrow}, t_{1}}$.

Finally, given $t \in \mathbb{R}$ small enough, we denote by $\mathfrak{D}_{\tau, f, t}^{+}$the surface $\mathfrak{D}_{\tau, f, t}^{+}$translated in the vertical direction by $\left(2-c^{\downarrow}+d^{\downarrow}-c^{\uparrow}+d^{\uparrow}+t_{1}+t\right) e_{3}$ :

$$
\mathfrak{D}_{\tau, f, t}^{+}:=\mathfrak{D}_{\tau, f}^{+}+\left(2-c^{\downarrow}+d^{\downarrow}-c^{\uparrow}+d^{\uparrow}+t_{1}+t\right) e_{3} .
$$

This is a constant mean curvature surface which is symmetric with respect to the dihedral group $\operatorname{Dih}_{m+1}^{(2)}$. Observe that the boundary of $\mathfrak{D}_{\tau, f, t}^{+}$is close to the upper boundary of $\mathfrak{S}_{\tau_{1}, \rho_{1}, f_{1}^{\uparrow}, f_{1}^{\downarrow}, t_{1}}$.

To complete the proof of the main theorem, it remains to adjust the free parameters of our construction, namely, $t, t_{1}, \tau_{1}, \tau_{2}, \rho_{1} \in \mathbb{R}$, and the functions $f_{1}^{\downarrow}, f_{2}^{\downarrow}, f^{\dagger}$ and $f_{1}^{\uparrow}$ defined on $S^{1}$, so that

$$
\mathfrak{C}_{\tau_{2}, f_{2}, \rho_{1}}^{(0)} \sqcup \cdots \sqcup \mathfrak{C}_{\tau_{2}, f_{2}^{\downarrow}, \rho_{1}}^{(m)} \sqcup \mathfrak{S}_{\tau_{1}, \rho_{1}, f_{1}^{\downarrow}, f_{1}^{\downarrow}, t_{1}} \sqcup \mathfrak{D}_{\tau, f, t}^{+}
$$

constitute a $\mathscr{C}^{1}$ surface which can be extended by reflection through the horizontal plane as a $\mathscr{C}^{1}$ surface which is complete, noncompact, and has two ends of Delaunay type (asymptotic to a nodoid end). Observe that the surface is invariant under the action of the dihedral group $\left.\operatorname{Dih}^{(3}\right)_{m+1}$ and that there is still one free parameter, namely, $\tau$, which determines the Delaunay type end and hence the vertical flux of the surface.

This surface is in fact piecewise smooth and has constant mean curvature equal to 1 away from the boundaries where the connected sum is performed. Since all pieces have constant mean curvature identically equal to 1 , elliptic regularity theory then implies that this surface is in fact a smooth surface. Indeed, near one of the boundaries where the connected sum is performed the surface is a graph of a 
function, say $u^{\text {ins }}$ defined over $A_{\tau}^{\text {ins }}$ and another function, say $u^{\text {out }} \operatorname{defined~over~} A_{\tau}^{\text {out }}$. The functions $u^{\text {ins }}$ and $u^{\text {out }}$ are $\mathscr{C}^{2, \alpha}$ and solve the mean curvature equation

$$
\frac{1}{2} \operatorname{div}\left(\frac{\nabla u}{\sqrt{1+|\nabla u|^{2}}}\right)=1
$$

on their respective domains of definition (for the sake of simplicity, we assume that the mean curvature vector is upward pointing near the boundary we are interested in). Moreover, $u^{\text {ins }}=u^{\text {out }}$ and $\partial_{r} u^{\text {ins }}=\partial_{r} u^{\text {out }}$ on $\partial A_{\tau}^{\text {ins }} \cap \partial A_{\tau}^{\text {out }}$. This implies that the function $u$ defined on $A_{\tau}^{\text {ins }} \cup A_{\tau}^{\text {out }}$ by $u:=u^{\text {ins }}$ on $A_{\tau}^{\text {ins }}$ and $u:=u^{\text {out }}$ on $A_{\tau}^{\text {out }}$ belongs to $\mathscr{C}^{1,1}$ and is a weak solution of (7-1) on $A_{\tau}^{\text {ins }} \cup A_{\tau}^{\text {out }}$. Elliptic regularity implies that $u$ is $\mathscr{C}^{2, \alpha}$, and hence the surface we have obtained is a smooth constant mean curvature surface.

Therefore, to complete the proof, it remains to explain how to find the parameters $t, t_{1}, \tau_{1}, \tau_{2}, \rho_{1} \in \mathbb{R}$, and the functions $f_{1}^{\downarrow}, f_{2}^{\downarrow}, f^{\uparrow}$, and $f_{1}^{\uparrow}$ defined on $S^{1}$, so that the following system of equations on $S^{1}$ is fulfilled:

$$
\begin{array}{ccc}
U^{\uparrow}-c^{\uparrow}+t=V^{\uparrow}-1-d^{\uparrow} & \text { and } & \partial_{r}\left(V^{\uparrow}-U^{\uparrow}\right)=0, \\
V^{\downarrow}+1-c^{\downarrow}+t_{1}=U^{\downarrow}-d^{\downarrow} & \text { and } & \partial_{r}\left(V^{\downarrow}-U^{\downarrow}\right)=0 .
\end{array}
$$

Recall that, even though this is not apparent in the notations, all functions and constants depend on the parameters and boundary data. The rest of this section is devoted to the proof that the above system does have a solution, provided $\tau$ is small enough.

Proposition 7.1. There exists $\kappa>0$ such that, for all $\tau>0$ small enough, there exist parameters $t, t_{1}, \tau_{1}, \tau_{2}, \rho_{1}$, and functions $f_{1}^{\downarrow}, f_{2}^{\downarrow}, f^{\dagger}, f_{1}^{\downarrow}$ defined on $S^{1}$ and satisfying the above symmetries and estimates such that the system (7-2) and (7-3) is satisfied.

Proof. First we make use of Propositions 4.12, 5.5, and 6.7 to get the expansion of the functions $U^{\uparrow}, V^{\uparrow}, U^{\downarrow}$ and $V^{\downarrow}$. Recalling that we have to restrict all those functions to $S^{1}$, it is easy to check, using (4-16) and (6-9), that the first two equations of the system we have to solve read

$$
\begin{aligned}
& t+f_{1}^{\uparrow}-f^{\uparrow}=\bar{V}^{\uparrow}-\bar{U}^{\uparrow}, \\
& \left(\tau_{1}-\tau\right)+\partial_{r}\left(W_{f_{1}^{\uparrow}}^{\text {out }}-W_{f^{\uparrow}}^{\text {ins }}\right)=\partial_{r}\left(\bar{V}^{\uparrow}-\bar{U}^{\uparrow}\right),
\end{aligned}
$$

while, using (5-7) and (6-12), we see that the next two equations are given by

$$
\begin{aligned}
& t_{1}-\tau^{3 / 4}\left(\rho_{1}-\frac{m}{m+1} \frac{\tau_{1}}{2 \rho_{1}}\right) z_{0} \cdot x+f_{1}^{\downarrow}-f_{2}^{\downarrow}=\bar{U}^{\downarrow}-\bar{V}^{\downarrow}, \\
& -\left(\frac{\tau_{1}}{m+1}-\tau_{2}\right)-\tau^{3 / 4}\left(\rho_{1}-\frac{m}{m+1} \frac{\tau_{1}}{2 \rho_{1}}\right) z_{0} \cdot x+\partial_{r}\left(W_{f_{1}^{\downarrow}}^{\text {out }}-W_{f_{2}^{\downarrow}}^{\text {ins }}\right) \\
& =\partial_{r}\left(\bar{U}^{\downarrow}-\bar{V}^{\downarrow}\right) .
\end{aligned}
$$


In writing this system one has to be a bit careful about the invariance of the functions we are interested in. Indeed, in (7-4), all functions are invariant under the action of $\operatorname{Dih}_{m+1}^{(2)}$, while in (7-5), all functions are invariant under the action of the symmetry $\mathscr{S}_{2}$.

Let us denote by $\Pi^{0}$ the $L^{2}\left(S^{1}\right)$-orthogonal projection over the space of constant functions, by $\Pi^{1}$, the $L^{2}\left(S^{1}\right)$-orthogonal projection over the space spanned by the function $x \mapsto z_{0} \cdot x$, and by $\Pi^{\perp}$, the $L^{2}\left(S^{1}\right)$-orthogonal projection over the orthogonal complement of the space spanned by the constant function and the function $x \mapsto z_{0} \cdot x$.

We project this system over the $L^{2}\left(S^{1}\right)$-orthogonal complement of the constant function and the function $x \mapsto z_{0} \cdot x$. We obtain the coupled system

$$
\begin{aligned}
& f_{1}^{\uparrow}-f^{\uparrow}=\Pi^{\perp}\left(\bar{V}^{\uparrow}-\bar{U}^{\uparrow}\right), \\
& \partial_{r}\left(W_{f_{1}^{\uparrow}}^{\text {out }}-W_{f^{\uparrow}}^{\text {ins }}\right)=\Pi^{\perp} \partial_{r}\left(\bar{V}^{\uparrow}-\bar{U}^{\uparrow}\right), \\
& f_{1}^{\downarrow, \perp}-f_{2}^{\downarrow}=\Pi^{\perp}\left(\bar{U}^{\downarrow}-\bar{V}^{\downarrow}\right), \\
& \partial_{r}\left(W_{f_{1}^{l, \perp}}^{\text {out }}-W_{f_{2}^{\downarrow}}^{\text {ins }}\right)=\Pi^{\perp} \partial_{r}\left(\bar{U}^{\downarrow}-\bar{V}^{\downarrow}\right),
\end{aligned}
$$

where we recall that we have decomposed $f_{1}^{\downarrow}=\lambda_{1} z_{0} \cdot x+f_{1}^{l, \perp}$.

The projection of the system (7-4)-(7-5) over the space of constant functions leads to the coupled system

$$
\begin{aligned}
& t=\Pi^{0}\left(\bar{V}^{\uparrow}-\bar{U}^{\urcorner}\right), \\
& \tau_{1}-\tau=\Pi^{0} \partial_{r}\left(\bar{V}^{\uparrow}-\bar{U}^{\urcorner}\right), \\
& t_{1}=\Pi^{0}\left(\bar{U}^{\downarrow}-\bar{V}^{\downarrow}\right), \\
& \tau_{2}-\frac{\tau_{1}}{m+1}=\Pi^{0} \partial_{r}\left(\bar{U}^{\downarrow}-\bar{V}^{\downarrow}\right) .
\end{aligned}
$$

Finally, the projection of the system (7-4)-(7-5) over the space of functions spanned by $x \mapsto z_{0} \cdot x$ leads to the coupled system

$$
\begin{aligned}
& \left(\lambda_{1}-\tau^{3 / 4}\left(\rho_{1}-\frac{m}{m+1} \frac{\tau_{1}}{2 \rho_{1}}\right)\right) z_{0} \cdot x=\Pi^{1}\left(\bar{U}^{\downarrow}-\bar{V} \downarrow\right), \\
& \left(-\lambda_{1}-\tau^{3 / 4}\left(\rho_{1}-\frac{m}{m+1} \frac{\tau_{1}}{2 \rho_{1}}\right)\right) z_{0} \cdot x=\Pi^{1} \partial_{r}\left(\bar{U}^{\downarrow}-\bar{V}^{\downarrow}\right) .
\end{aligned}
$$

To obtain the second equation, we have used the fact that

$$
W_{f_{1}^{\downarrow}}^{\text {out }}=\lambda_{1} \frac{z_{0} \cdot x}{|x|^{2}}+W_{f_{1}^{l, \perp}}^{\text {out }} \text {. }
$$

Observe that the right sides of (7-6), (7-7), and (7-8) do not depend on $t$ and $t_{1}$. Hence the first and third equations in (7-7) give us the values of $t$ and $t_{1}$, once the rest of the equations are solved. 
For all $\tau$ small enough, we solve (7-6) using a fixed-point theorem for contraction mappings to obtain a solution $\left(f^{\uparrow}, f_{1}^{\uparrow}, f_{1}^{\downarrow, \perp}, f_{2}^{\downarrow}\right)$ continuously depending on the parameters $\tau_{1}, \tau_{2}, \rho_{1}, \lambda_{1}$ (and $\tau$ ). Then we introduce the corresponding solution in (7-7) and (7-8) to get a nonlinear system in $\tau_{1}, \tau_{2}$, and $\rho_{1}$, which we solve using Browder's fixed-point theorem.

To begin with, we explain how (7-6) can be rewritten in diagonal form. This makes use of the following result, whose proof can be found, for example, in [Mazzeo and Pacard 2001]:

\section{Proposition 7.2. The operator}

$$
\mathscr{b}^{2, \alpha}\left(S^{1}\right)^{\perp} \ni f \mapsto \partial_{r}\left(W_{f}^{\text {ins }}-W_{f}^{\text {out }}\right)_{\mid r=1} \in \mathscr{b}^{1, \alpha}\left(S^{1}\right)^{\perp}
$$

is an isomorphism. Here $\mathfrak{b}^{k, \alpha}\left(S^{1}\right)^{\perp}$ denotes the image of $\mathfrak{C}^{k, \alpha}\left(S^{1}\right)$ under $\Pi^{\perp}$. Proof. The Fourier decomposition of a function $f \in \mathscr{C}^{k, \alpha}\left(S^{1}\right)^{\perp}$ is given by

$$
f(\theta)=\sum_{n \neq 0, \pm 1} f_{n} e^{i n \theta}
$$

in which case

$$
W_{f}^{\text {out }}=\sum_{n \neq 0, \pm 1} f_{n} r^{-|n|} e^{i n \theta} \quad \text { and } \quad W_{f}^{\text {ins }}=\sum_{n \neq 0, \pm 1} f_{n} r^{|n|} e^{i n \theta}
$$

Therefore,

$$
\partial_{r}\left(W_{f}^{\text {ins }}-W_{f}^{\text {out }}\right)_{\mid r=1}=2 \sum_{n \neq 0, \pm 1} f_{n}|n| e^{i n \theta}
$$

is equal to twice the Dirichlet to Neumann map for the Laplace operator in the unit disc. This is a well-defined, selfadjoint, first order elliptic operator which is injective, and elliptic regularity theory implies that it is an isomorphism.

Using this result, the system (7-6) can be rewritten as

$$
\left(f^{\uparrow}, f_{1}^{\uparrow}, f_{1}^{\downarrow, \perp}, f_{2}^{\downarrow}\right)=\mathbb{N}_{\tau_{1}, \tau_{2}, \rho_{1}, \lambda_{1}}^{\perp}\left(f^{\uparrow}, f_{1}^{\uparrow} f_{1}^{\downarrow, \perp}, f_{2}^{\downarrow}\right),
$$

where the nonlinear operator $\mathbb{N}_{\tau_{1}, \tau_{2}, \rho_{1}, \lambda_{1}}^{\perp}$ satisfies

$$
\left\|\mathbb{N}_{\tau_{1}, \tau_{2}, \rho_{1}, \lambda_{1}}^{\perp}\left(f^{\uparrow}, f_{1}^{\uparrow}, f_{1}^{\downarrow, \perp}, f_{2}^{\downarrow}\right)\right\|_{\left(\mathscr{C}^{2, \alpha}\left(S^{1}\right)\right)^{4}} \leq C \tau^{3 / 2}
$$

for some constant $C>0$ independent of $\kappa>0$, provided $\tau$ is chosen small enough. This last estimate follows directly from (4-17), (5-8) and (6-10). Moreover, thanks to (4-18), (5-9), and (6-11), provided $\kappa>0$ is fixed larger than the constant $C$ which appears in (7-9), we can use a fixed-point theorem for contraction mappings in the ball of radius $\kappa \tau^{3 / 2}$ in $\left(\Pi^{\perp} \mathscr{C}^{2, \alpha}\left(S^{1}\right)\right)^{4}$ to get the existence of a solution of (7-9) for all $\tau>0$ small enough. This solution depends continuously on $\tau_{1}, \tau_{2}$, $\rho_{1}$, and $\lambda_{1}$, since $\mathbb{N}_{\tau_{1}, \tau_{2}, \rho_{1}, \lambda_{1}}^{\perp}$ does (observe that $\mathbb{N}_{\tau_{1}, \tau_{2}, \rho_{1}, \lambda_{1}}^{\perp}$ depends implicitly on 
$\tau)$. We now insert this solution in (7-7) and (7-8). With simple manipulations, we conclude that it remains to solve the nonlinear system

$$
\left(\tau_{1}-\tau, \tau_{2}-\frac{\tau}{m+1}, \tau^{3 / 4}\left(\rho_{1}-\frac{m}{m+1} \frac{\tau}{2 \rho_{1}}\right), \lambda_{1}\right)=\mathbb{N}^{0}\left(\tau_{1}, \tau_{2}, \rho_{1}, \lambda_{1}\right),
$$

where $\mathbb{N}^{0}$ satisfies

$$
\left\|\mathbb{N}^{0}\left(\tau_{1}, \tau_{2}, \rho_{1}, \lambda_{1}\right)\right\|_{\mathbb{R}^{4}} \leq C \tau^{3 / 2}
$$

for some constant $C>0$ independent of $\kappa>0$, provided $\tau$ is chosen small enough. Moreover, $\mathbb{N}^{0}$ depends continuously on the parameters $\tau_{1}, \tau_{2}, \rho_{1}$, and $\lambda_{1}$ (observe that $\mathbb{N}^{0}$ depends implicitly on $\tau$ ). Equation (7-10) can then be solved using a simple degree argument (Browder's fixed-point theorem).

\section{Appendix}

We discuss the elementary result in the theory of second order ordinary differential equations which is used at the end of the proof of Proposition 4.8. Assume that we are given a function $s \mapsto p(s)$ which is periodic (say of period $S>0$ ). Further assume that the homogeneous problem $\left(\partial_{s}^{2}+p\right) w^{+}=0$ has a nontrivial periodic solution of period $S$. Without loss of generality, we can assume that $w^{+}(0)=1$ and $\partial_{s} w^{+}(0)=0$ (just choose the origin so that 0 coincides with a point where $w^{+}$ achieves its maximum). Let $w^{-}$be the unique solution of $\left(\partial_{s}^{2}+p\right) w^{-}=0$ such that $w^{-}(0)=0$ and $\partial_{s} w^{-}(0)=1$. The Wronskian of $w^{+}$and $w^{-}$being constant, we conclude that

$$
\begin{aligned}
\partial_{s} w^{-}(S) & =\partial_{s} w^{-}(S) w^{+}(S)-\partial_{s} w^{+}(S) w^{-}(S) \\
& =\partial_{s} w^{-}(0) w^{+}(0)-\partial_{s} w^{+}(0) w^{-}(0) \\
& =1
\end{aligned}
$$

We define

$$
v(s):=w^{-}(S+s)-w^{-}(S) w^{+}(s) .
$$

It is clear that $v$ is a solution of $\left(\partial_{s}^{2}+p\right) v=0$. Further, observe that $\partial_{s} v(0)=1$ and $v(0)=0$. Therefore, $v=w^{-}$. This proves that

$$
w^{-}(S+s)=w^{-}(s)+w^{-}(S) w^{+}(s),
$$

and hence $w^{-}$is at most growing linearly in the sense that $\left|w^{-}(s)\right| \leq C(1+|s|)$ for some constant $C>0$.

\section{References}

[Colding and Minicozzi 2011] T. H. Colding and W. P. Minicozzi, II, A course in minimal surfaces, Graduate Studies in Mathematics 121, American Mathematical Society, Providence, RI, 2011. MR $2780140 \mathrm{Zbl} 1242.53007$ 
[Eells 1987] J. Eells, “The surfaces of Delaunay", Math. Intelligencer 9:1 (1987), 53-57. MR 88h: 53011 Zbl 0605.53002

[Fakhi and Pacard 2000] S. Fakhi and F. Pacard, "Existence result for minimal hypersurfaces with a prescribed finite number of planar ends", Manuscripta Math. 103:4 (2000), 465-512. MR 2002d:53017 Zbl 0992.53011

[Hauswirth and Pacard 2007] L. Hauswirth and F. Pacard, "Higher genus Riemann minimal surfaces", Invent. Math. 169:3 (2007), 569-620. MR 2008i:58013 Zbl 1129.53009

[Lawson 1977] H. B. Lawson, Jr., Lectures on minimal submanifolds, I, Monografías de Matemática 14, Instituto de Matemática Pura e Aplicada, Rio de Janeiro, 1977. MR 82d:53035a Zbl 0924.60012

[Lockhart and McOwen 1985] R. B. Lockhart and R. C. McOwen, "Elliptic differential operators on noncompact manifolds", Ann. Scuola Norm. Sup. Pisa Cl. Sci. (4) 12:3 (1985), 409-447. MR 87k:58266 Zbl 0615.58048

[Mazzeo 1991] R. Mazzeo, "Elliptic theory of differential edge operators, I", Comm. Partial Differential Equations 16:10 (1991), 1615-1664. MR 93d:58152 Zbl 0745.58045

[Mazzeo and Pacard 2001] R. Mazzeo and F. Pacard, "Constant mean curvature surfaces with Delaunay ends”, Comm. Anal. Geom. 9:1 (2001), 169-237. MR 2002b:53005 Zbl 1005.53006

[Mazzeo et al. 2001] R. Mazzeo, F. Pacard, and D. Pollack, "Connected sums of constant mean curvature surfaces in Euclidean 3 space", J. Reine Angew. Math. 536 (2001), 115-165. MR 2002d:53020 Zbl 0972.53010

[Mazzeo et al. 2005] R. Mazzeo, F. Pacard, and D. Pollack, "The conformal theory of Alexandrov embedded constant mean curvature surfaces in $\mathbb{R}^{3}$ ", pp. 525-559 in Global theory of minimal surfaces, edited by D. Hoffman, Clay Math. Proc. 2, Amer. Math. Soc., Providence, RI, 2005. MR 2006g:53009 Zbl 1101.53006

[Melrose 1993] R. B. Melrose, The Atiyah-Patodi-Singer index theorem, Research Notes in Mathematics 4, A K Peters Ltd., Wellesley, MA, 1993. MR 96g:58180 Zbl 0796.58050

[Pacard 2008] F. Pacard, "Connected sum constructions in geometry and nonlinear analysis", Unpublished lecture notes, 2008, Available at http://www.math.polytechnique.fr/ pacard/Publications/ Lecture-Part-I.pdf.

[Rossman 2005] W. Rossman, "The first bifurcation point for Delaunay nodoids", Experiment. Math. 14:3 (2005), 331-342. MR 2006j:53011 Zbl 1092.53010

Received July 31, 2012. Revised December 16, 2012.

FRANK PACARD

Centre de Mathématiques Laurent Schwartz, UMR-CNRS 7640

ÉCOLE POLYTECHNIQUe

Palaiseau, 91128

FRANCE

frank.pacard@math.polytechnique.fr

HAROLD ROSENBERG

Instituto de Matemática Pura e APlicada

110 ESTRADA DONA CASTORINA

RIO DE JANEIRO 22460-320

BRAZIL

hrosen@free.fr 


\title{
PACIFIC JOURNAL OF MATHEMATICS
}

\author{
msp.org/pjm
}

Founded in 1951 by E. F. Beckenbach (1906-1982) and F. Wolf (1904-1989)

\section{EDITORS}

V. S. Varadarajan (Managing Editor)

Department of Mathematics

University of California

Los Angeles, CA 90095-1555

pacific@math.ucla.edu

Paul Balmer

Department of Mathematics

University of California

Los Angeles, CA 90095-1555

balmer@math.ucla.edu

Daryl Cooper

Department of Mathematics

University of California

Santa Barbara, CA 93106-3080 cooper@math.ucsb.edu

Jiang-Hua $\mathrm{Lu}$

Department of Mathematics

Pokfulam Rd., Hong Kong jhlu@maths.hku.hk
The University of Hong Kong

Don Blasius

Department of Mathematics University of California

Los Angeles, CA 90095-1555

blasius@math.ucla.edu

Robert Finn

Department of Mathematics Stanford University

Stanford, CA 94305-2125

finn@math.stanford.edu

Sorin Popa

Department of Mathematics

University of California

Los Angeles, CA 90095-1555 popa@math.ucla.edu

Paul Yang

Department of Mathematics Princeton University

Princeton NJ 08544-1000

yang@math.princeton.edu

\section{PRODUCTION}

Silvio Levy, Scientific Editor, production@msp.org

\section{SUPPORTING INSTITUTIONS}

ACADEMIA SINICA, TAIPEI

CALIFORNIA INST. OF TECHNOLOGY

INST. DE MATEMÁTICA PURA E APLICADA

KEIO UNIVERSITY

MATH. SCIENCES RESEARCH INSTITUTE

NEW MEXICO STATE UNIV.

OREGON STATE UNIV.

\author{
STANFORD UNIVERSITY \\ UNIV. OF BRITISH COLUMBIA \\ UNIV. OF CALIFORNIA, BERKELEY \\ UNIV. OF CALIFORNIA, DAVIS \\ UNIV. OF CALIFORNIA, LOS ANGELES \\ UNIV. OF CALIFORNIA, RIVERSIDE \\ UNIV. OF CALIFORNIA, SAN DIEGO \\ UNIV. OF CALIF., SANTA BARBARA
}

\author{
Vyjayanthi Chari \\ Department of Mathematics \\ University of California \\ Riverside, CA 92521-0135 \\ chari@math.ucr.edu \\ Kefeng Liu \\ Department of Mathematics \\ University of California \\ Los Angeles, CA 90095-1555 \\ liu@math.ucla.edu \\ Jie Qing \\ Department of Mathematics \\ University of California \\ Santa Cruz, CA 95064 \\ qing@cats.ucsc.edu
}

These supporting institutions contribute to the cost of publication of this Journal, but they are not owners or publishers and have no responsibility for its contents or policies.

See inside back cover or msp.org/pjm for submission instructions.

The subscription price for 2013 is US \$400/year for the electronic version, and \$485/year for print and electronic.

Subscriptions, requests for back issues and changes of subscribers address should be sent to Pacific Journal of Mathematics, P.O. Box 4163, Berkeley, CA 94704-0163, U.S.A. The Pacific Journal of Mathematics is indexed by Mathematical Reviews, Zentralblatt MATH, PASCAL CNRS Index, Referativnyi Zhurnal, Current Mathematical Publications and the Science Citation Index.

The Pacific Journal of Mathematics (ISSN 0030-8730) at the University of California, c/o Department of Mathematics, 798 Evans Hall \#3840, Berkeley, CA 94720-3840, is published twelve times a year. Periodical rate postage paid at Berkeley, CA 94704, and additional mailing offices. POSTMASTER: send address changes to Pacific Journal of Mathematics, P.O. Box 4163, Berkeley, CA 94704-0163.

PJM peer review and production are managed by EditFLOW ${ }^{\circledR}$ from Mathematical Sciences Publishers.

\section{PUBLISHED BY}

mathematical sciences publishers

nonprofit scientific publishing

http://msp.org/

(C) 2013 Mathematical Sciences Publishers 


\section{PACIFIC JOURNAL OF MATHEMATICS}

Volume $266 \quad$ No. $1 \quad$ November 2013

Multiplicity of solutions to the Yamabe problem on collapsing $\quad 1$ Riemannian submersions

Renato G. Bettiol and Paolo Piccione

Rank gradient of small covers

DARLAN GIRÃO

Nonrationality of nodal quartic threefolds

KYUSIK HONG

Supertropical linear algebra

ZUR IZHAKIAN, MANFRED KNEBUSCH and LOUIS ROWEN

Isometry groups among topological groups

PIOTR NIEMIEC

Singularities and Liouville theorems for some special conformal

Hessian equations

QIANZHONG OU

Attaching handles to Delaunay nodoids

FRANK PACARD and HAROLD ROSENBERG

Some new canonical forms for polynomials

BRUCE REZNICK

Applications of the deformation formula of holomorphic one-forms 